\title{
A global fit of the MSSM with GAMBIT
}

\author{
The GAMBIT Collaboration: Peter Athron ${ }^{1,2, a}$, Csaba Balázs ${ }^{1,2}$, Torsten Bringmann ${ }^{3}$, Andy Buckley ${ }^{4}$, \\ Marcin Chrzaszcz $^{5,6}$, Jan Conrad ${ }^{7,8}$, Jonathan M. Cornell ${ }^{9}$, Lars A. Dal ${ }^{3}$, Joakim Edsjö ${ }^{7,8}$, Ben Farmer ${ }^{7,8}$, \\ Paul Jackson $^{2,10}$, Abram Krislock ${ }^{3}$, Anders Kvellestad ${ }^{11, b}$, Farvah Mahmoudi ${ }^{12,13, e}$, Gregory D. Martinez ${ }^{14}$, \\ Antje Putze ${ }^{15}$, Are Raklev ${ }^{3}$, Christopher Rogan ${ }^{16}$, Aldo Saavedra ${ }^{2,17}$, Christopher Savage ${ }^{11}$, Pat Scott ${ }^{18, c}$, \\ Nicola Serra ${ }^{5}$, Christoph Weniger ${ }^{19}$, Martin White ${ }^{2,10, d}$ \\ ${ }^{1}$ School of Physics and Astronomy, Monash University, Melbourne, VIC 3800, Australia \\ ${ }^{2}$ Australian Research Council Centre of Excellence for Particle Physics at the Tera-Scale, Australia, http://www.coepp.org.au/ \\ ${ }^{3}$ Department of Physics, University of Oslo, 0316 Oslo, Norway \\ ${ }^{4}$ SUPA, School of Physics and Astronomy, University of Glasgow, Glasgow G12 8QQ, UK \\ ${ }^{5}$ Physik-Institut, Universität Zürich, Winterthurerstrasse 190, 8057 Zurich, Switzerland \\ ${ }^{6}$ H. Niewodniczański Institute of Nuclear Physics, Polish Academy of Sciences, 31-342 Kraków, Poland \\ ${ }^{7}$ Oskar Klein Centre for Cosmoparticle Physics, AlbaNova University Centre, 10691 Stockholm, Sweden \\ ${ }^{8}$ Department of Physics, Stockholm University, 10691 Stockholm, Sweden \\ ${ }^{9}$ Department of Physics, McGill University, 3600 rue University, Montreal, QC H3A 2T8, Canada \\ ${ }^{10}$ Department of Physics, University of Adelaide, Adelaide, SA 5005, Australia \\ 11 NORDITA, Roslagstullsbacken 23, 10691 Stockholm, Sweden \\ ${ }^{12}$ Univ Lyon, Univ Lyon 1, ENS de Lyon, CNRS, Centre de Recherche Astrophysique de Lyon UMR5574, 69230 Saint-Genis-Laval, France \\ 13 Theoretical Physics Department, CERN, 1211 Geneva 23, Switzerland \\ 14 Physics and Astronomy Department, University of California, Los Angeles, CA 90095, USA \\ ${ }^{15}$ LAPTh, Université de Savoie, CNRS, 9 chemin de Bellevue, B.P. 110, 74941 Annecy-le-Vieux, France \\ ${ }^{16}$ Department of Physics, Harvard University, Cambridge, MA 02138, USA \\ ${ }^{17}$ Centre for Translational Data Science, Faculty of Engineering and Information Technologies, School of Physics, The University of Sydney, \\ Sydney, NSW 2006, Australia \\ 18 Department of Physics, Blackett Laboratory, Imperial College London, Prince Consort Road, London SW7 2AZ, UK \\ ${ }^{19}$ GRAPPA, Institute of Physics, University of Amsterdam, Science Park 904, 1098 XH Amsterdam, The Netherlands
}

\begin{abstract}
We study the seven-dimensional Minimal Supersymmetric Standard Model (MSSM7) with the new GAMBIT software framework, with all parameters defined at the weak scale. Our analysis significantly extends previous weak-scale, phenomenological MSSM fits, by adding more and newer experimental analyses, improving the accuracy and detail of theoretical predictions, including dominant uncertainties from the Standard Model, the Galactic dark matter halo and the quark content of the nucleon, and employing novel and highly-efficient statistical sampling methods to scan the parameter space. We find regions of the MSSM7
\end{abstract}

\footnotetext{
a e-mail: peter.athron@coepp.org.au

b e-mail: anders.kvellestad@nordita.org

c e-mail: p.scott@imperial.ac.uk

d e-mail: martin.white@adelaide.edu.au

e Also Institut Universitaire de France, 103 boulevard Saint-Michel, 75005 Paris, France.
}

that exhibit co-annihilation of neutralinos with charginos, stops and sbottoms, as well as models that undergo resonant annihilation via both light and heavy Higgs funnels. We find high-likelihood models with light charginos, stops and sbottoms that have the potential to be within the future reach of the LHC. Large parts of our preferred parameter regions will also be accessible to the next generation of direct and indirect dark matter searches, making prospects for discovery in the near future rather good.

\section{Contents}

1 Introduction ................ 2

2 Models and scanning framework . . . . . . . . . . 3

2.1 Model definitions and parameters . . . . . . . . 3

2.1.1 MSSM7 . . . . . . . . . . 3

2.1.2 Nuisance parameters . . . . . . . . 3

2.2 Scanning methodology . . . . . . . . . . . 4 
3 Observables and likelihoods ........... 5

4 Results ................. . 6

4.1 Best fits . . . . . . . . . . . 6

4.2 Preferred regions . . . . . . . . . . 9

5 Future prospects .............. . 14

5.1 LHC . . . . . . . . . . . . . . . . 14

5.2 Direct detection of dark matter . . . . . . . . 18

5.3 Indirect detection of dark matter . . . . . . . . 19

6 Conclusions ............... . 20

References................ 21

\section{Introduction}

The most straightforward supersymmetric extension of the Standard Model (SM) of elementary particles is the Minimal Supersymmetric Standard Model (MSSM) [1]. The MSSM can help deal with many of the theoretical and experimental shortcomings of the SM. Most notably it stabilises the electroweak scale [2-6] with respect to large corrections from new physics at the Planck scale [7-11], allows the unification of gauge couplings [12-15], provides a dark matter (DM) candidate that can fit the observed relic abundance [16,17] and predicts a light Higgs boson, in accordance with the 2012 discovery $[18,19]$. This has prompted a vast number of investigations of this model, with recent literature studying precision corrections to the Higgs mass [20-29] and other aspects of Higgs physics [30-40], DM [41-78], the matter-antimatter asymmetry of the Universe [79-81], vacuum stability [82], cosmic inflation [83-90], and various measurements of precision [91-98] and flavour [99, 100] observables. MSSM predictions for observations related to the above also yield the most important constraints on the theory.

Indeed the MSSM is one of the best-studied extensions to the SM to date. The latest literature covers topics ranging from global fits to the MSSM with different choices for the number of dimensions in the electroweak scale parameterisation [101-107], to studies of various aspects of its more constrained versions defined at a high scale [108-118]. Theoretical considerations, such as naturalness [119-130], and gauge unification within the MSSM [131-135], have also been a concern. Due to the steady stream of results from the LHC, the implications of collider searches for the MSSM have been a particularly active field of study [136-159].

The most general version of the MSSM has a very large number of parameters. Assuming only $C P$ conservation, there are in total 63 free parameters, coming mostly from soft supersymmetry-breaking terms. There are two distinct ways to approach the exploration of the MSSM. The first is to take inspiration from the apparent unification of the gauge couplings at a high scale, defining a small set of unified parameters at that scale - a Grand Unified Theory (GUT) - and then run them down to the electroweak scale in order to compare with experiment. This is done for example in the four-parameter constrained MSSM (CMSSM) with common mass parameters for the scalar and fermion soft masses [160], and various generalisations of the CMSSM, such as the non-universal Higgs mass models 1 and 2 (NUHM1 and NUHM2), which split the Higgs soft masses from the other scalar masses [161-165]. We have carried out extensive global fits to these GUT-motivated models in a companion paper to this one [166].

The other approach is to remain agnostic about the highscale properties of the theory, and to define all the parameters at an energy near the electroweak scale. This is the socalled 'phenomenological MSSM' (pMSSM [167]). Given the impracticality of studying the complete parameter space, it is necessary to make some physically-motivated assumptions and simplifications in order to focus upon a relevant and tractable lower-dimensional subspace of the full model.

In this paper, we perform the first global fit of the weakscale phenomenological MSSM to make use of the GAMBIT global-fitting framework [168]. Our work improves upon previous pMSSM studies on the following fronts:

1. The larger number of observables in our composite likelihood function, including: sparticle searches at LEP, Run I and Run II of the LHC, observables and constraints on the Higgs sector from LEP, the Tevatron and the LHC, direct and indirect dark matter searches from a multitude of experiments, and a large number of flavour and precision observables.

2. Experimental likelihoods reconstructed from event rates, where applicable, including: Monte Carlo simulation of LHC observables, kinematical acceptance-based event rates for LEP sparticle searches, indirect DM search likelihoods computed at the level of single events, and direct DM search limits based on sophisticated simulation of the relevant experiments.

3. The use of the GAMBIT hierarchical model database and statistical framework, for an advanced treatment of uncertainties from dominant SM nuisance parameters across different observables, and a consistent theoretical and statistical treatment of all likelihoods.

4. The use of Diver [169], a new scanner based on differential evolution, which improves sampling performance compared to techniques used previously, and allows us to more accurately locate the high-likelihood regions.

5. The results in this paper are based on a public, opensource software, which allows for the reproduction and extension of our results by the interested reader. ${ }^{1}$

We begin in Sect. 2 by introducing the models, parameters and nuisances over which we scan, followed by our adopted

\footnotetext{
${ }^{1}$ http://gambit.hepforge.org.
} 
priors and sampling methodology. In Sect. 3, we then give a brief summary of the observables and likelihoods that we employ. We present our main results in Sect. 4 and their implications for future searches for the MSSM in Sect. 5, and conclude in Sect. 6.

All GAMBIT input files, generated likelihood samples and best-fit benchmarks for this paper are publicly available online through Zenodo [170].

\section{Models and scanning framework}

\subsection{Model definitions and parameters}

GAMBIT makes no fundamental distinction between parameters of BSM theories and nuisance parameters, scanning over each on an equal footing. Here we sample simultaneously from four different models: a 7-parameter phenomenological MSSM, and three models describing constraints on different areas of known physics relevant for calculating observables in the MSSM. These nuisance models respectively describe the SM, the Galactic DM halo, and nuclear matrix elements for different light quark flavours (relevant for direct detection of DM).

\subsubsection{MSSM7}

The most general formulation of the $C P$-conserving MSSM is given by the GAMBIT model MSSM63atQ. Full details of the Lagrangian can be found in Sec. 5.4.3 of Ref. [168]. This model has 63 free, continuous MSSM parameters: 3 gaugino masses $M_{1}, M_{2}$ and $M_{3}, 9$ parameters each from the trilinear coupling matrices $\mathbf{A}_{u}, \mathbf{A}_{d}$ and $\mathbf{A}_{e}, 6$ real numbers associated with each of the matrices of squared soft masses $\mathbf{m}_{Q}^{2}, \mathbf{m}_{u}^{2}, \mathbf{m}_{d}^{2}, \mathbf{m}_{L}^{2}$ and $\mathbf{m}_{e}^{2}$, and three additional parameters describing the Higgs sector. We choose to work with the explicit mass terms $m_{H_{u}}^{2}$ and $m_{H_{d}}^{2}$ for the two Higgs doublets. By swapping the Higgs bilinear couplings $b$ and $\mu$ for the ratio of vacuum expectation values for the up-type and downtype Higgs fields $\tan \beta \equiv v_{\mathrm{u}} / v_{\mathrm{d}}$, and demanding that the model successfully effect Electroweak Symmetry Breaking, we can reduce the remaining continuous freedom to a single parameter $(\tan \beta)$. This leaves only a free sign for $\mu$, which constitutes an additional (64th) discrete parameter. In this definition, $\tan \beta$ is specified at the scale $m_{Z}$, and all other parameters are defined at some other generic scale $Q$, usually taken to be near to the weak scale.

This parameter set is currently too large to explore in a global fit, and in any case much of the phenomenology can be captured in smaller models that incorporate simplifying assumptions. In this first paper, we explore the MSSM7atQ, a 7-parameter subspace of the MSSM63atQ. Inspired by GUT theories, we set
Table 1 MSSM7 parameters, ranges and priors adopted in the scans of this paper. For a parameter $x$ of mass dimension $n$, the "hybrid" prior is flat where $|x|<(100 \mathrm{GeV})^{n}$, and logarithmic elsewhere. The "split hybrid" prior for $M_{2}$ refers to the fact that we carried out every scan twice: once with a hybrid prior over $0 \leq M_{2} \leq 10 \mathrm{TeV}$, and again with a hybrid prior over $-10 \mathrm{TeV} \leq M_{2} \leq 0$. In addition to the priors listed here, we also carry out additional scans of fine-tuned regions associated with specific relic density mechanisms, where we restrict models to mass spectra that satisfy various conditions. See text for details

\begin{tabular}{llll}
\hline Parameter & Minimum & Maximum & Priors \\
\hline$A_{u_{3}}(Q)$ & $-10 \mathrm{TeV}$ & $10 \mathrm{TeV}$ & Flat, hybrid \\
$A_{d_{3}}(Q)$ & $-10 \mathrm{TeV}$ & $10 \mathrm{TeV}$ & Flat, hybrid \\
$M_{H_{u}}^{2}(Q)$ & $-(10 \mathrm{TeV})^{2}$ & $(10 \mathrm{TeV})^{2}$ & Flat, hybrid \\
$M_{H_{d}}^{2}(Q)$ & $-(10 \mathrm{TeV})^{2}$ & $(10 \mathrm{TeV})^{2}$ & Flat, hybrid \\
$m_{\tilde{f}}^{2}(Q)$ & 0 & $(10 \mathrm{TeV})^{2}$ & Flat, hybrid \\
$M_{2}(Q)$ & $-10 \mathrm{TeV}$ & $10 \mathrm{TeV}$ & Split; flat, hybrid \\
$\tan \beta\left(m_{Z}\right)$ & 3 & 70 & Flat \\
$\operatorname{sgn}(\mu)$ & + & & Fixed \\
$Q$ & $1 \mathrm{TeV}$ & & Fixed \\
\hline
\end{tabular}

$\frac{3}{5} \cos ^{2} \theta_{\mathrm{W}} M_{1}=\sin ^{2} \theta_{\mathrm{W}} M_{2}=\frac{\alpha}{\alpha_{\mathrm{s}}} M_{3}$,

at the scale $Q$. We assume that all entries in $\mathbf{A}_{u}, \mathbf{A}_{d}$ and $\mathbf{A}_{e}$ are zero except for $\left(\mathbf{A}_{u}\right)_{33}=A_{u_{3}}$ and $\left(\mathbf{A}_{d}\right)_{33}=A_{d_{3}}$. We take all of the off-diagonal entries in $\mathbf{m}_{Q}^{2}, \mathbf{m}_{u}^{2}, \mathbf{m}_{d}^{2}, \mathbf{m}_{L}^{2}$ and $\mathbf{m}_{e}^{2}$ to be zero, so as to suppress flavour-changing neutral currents. By setting all remaining mass matrix entries to a universal squared sfermion mass $m_{\tilde{f}}^{2}$, we reduce the final list of free parameters to $M_{2}, A_{u_{3}}, A_{d_{3}}, m_{\tilde{f}}^{2}, m_{H_{u}}^{2}, m_{H_{d}}^{2}$ and $\tan \beta$ (plus the input scale $Q$ and the sign of $\mu$ ). The MSSM7 has been studied in significant work in the previous literature, e.g. [171-176].

We assume that $R$-parity is conserved, making the lightest supersymmetric particle (LSP) absolutely stable, and discard all parameter combinations where the LSP is not a neutralino. This choice is discussed in more detail in Sec. 2.1.1 of the companion paper [166].

In Table 1, we give the parameter ranges over which we scan the MSSM7 in this paper. We choose to define all parameters other than $\tan \beta$ at $Q=1 \mathrm{TeV}$, and investigate positive $\mu$ (for a definition of $\mu$ please see the superpotential given in Sec. 5.4.3 of Ref. [168].). We intend to return to the $\mu<0$ branch of this model in future work, where we compare with less constrained subspaces of the MSSM63atQ.

\subsubsection{Nuisance parameters}

We make use of three different nuisance models in our scans: the SM as defined in SLHA2 [168,177], a model of the Galactic DM halo that follows a truncated Maxwell- 
Table 2 Standard Model, dark matter halo and nuclear nuisance parameters and ranges. We vary each of the parameters in the first section of the table simultaneously with MSSM7 parameters in all of our scans, employing flat priors on each. The parameters listed in the second section of the table are constant in all scans. Further details and references for the chosen values can be found in Sects. 2.1.2-2.1.4 of the companion paper [166]

\begin{tabular}{|c|c|c|}
\hline Parameter & & Value ( \pm range) \\
\hline \multicolumn{3}{|l|}{ Varied } \\
\hline Strong coupling & $\alpha_{s}^{\overline{M S}}\left(m_{Z}\right)$ & $0.1185(18)$ \\
\hline Top quark pole mass & $m_{t}$ & $173.34(2.28) \mathrm{GeV}$ \\
\hline Local DM density & $\rho_{0}$ & $0.2-0.8 \mathrm{GeV} \mathrm{cm}^{-3}$ \\
\hline Nuclear matrix el. (strange) & $\sigma_{s}$ & $43(24) \mathrm{MeV}$ \\
\hline Nuclear matrix el. (up + down) & $\sigma_{l}$ & $58(27) \mathrm{MeV}$ \\
\hline \multicolumn{3}{|l|}{ Fixed } \\
\hline Electromagnetic coupling & $1 / \alpha^{\overline{M S}}\left(m_{Z}\right)$ & 127.940 \\
\hline Fermi coupling $\times 10^{5}$ & $G_{F, 5}$ & 1.1663787 \\
\hline$Z$ pole mass & $m_{Z}$ & $91.1876 \mathrm{GeV}$ \\
\hline Bottom quark mass & $m_{b}^{\overline{M S}}\left(m_{b}\right)$ & $4.18 \mathrm{GeV}$ \\
\hline Charm quark mass & $m_{c}^{\overline{M S}}\left(m_{c}\right)$ & $1.275 \mathrm{GeV}$ \\
\hline Strange quark mass & $m_{s}^{\overline{M S}}(2 \mathrm{GeV})$ & $95 \mathrm{MeV}$ \\
\hline Down quark mass & $m_{d}^{\overline{M S}}(2 \mathrm{GeV})$ & 4.80 MeV \\
\hline Up quark mass & $m_{u}^{\overline{M S}}(2 \mathrm{GeV})$ & $2.30 \mathrm{MeV}$ \\
\hline$\tau$ pole mass & $m_{\tau}$ & $1.77682 \mathrm{GeV}$ \\
\hline \multirow[t]{4}{*}{ CKM Wolfenstein parameters } & $\lambda$ & 0.22537 \\
\hline & $A$ & 0.814 \\
\hline & $\bar{\rho}$ & 0.117 \\
\hline & $\bar{\eta}$ & 0.353 \\
\hline Most probable halo speed & $v_{0}$ & $235 \mathrm{~km} \mathrm{~s}^{-1}$ \\
\hline Local disk circular velocity & $v_{\text {rot }}$ & $235 \mathrm{~km} \mathrm{~s}^{-1}$ \\
\hline Local escape velocity & $v_{\mathrm{esc}}$ & $550 \mathrm{~km} \mathrm{~s}^{-1}$ \\
\hline Up contribution to proton spin & $\Delta_{u}^{(p)}$ & 0.842 \\
\hline Down contrib. to proton spin & $\Delta_{d}^{(p)}$ & -0.427 \\
\hline Strange contrib. to proton spin & $\Delta_{s}^{(p)}$ & -0.085 \\
\hline
\end{tabular}

Boltzmann velocity distribution [168, 178], and a model containing nuclear matrix elements required for calculating DMnucleon couplings $[168,178]$. We vary a total of five parameters across these models: the strong coupling at scale $m_{Z}$ in the $\overline{M S}$ scheme, the top pole mass, the local density of DM, and the strange and up/down quark contents of the nucleon. The treatment of these models and parameters here is identical to the treatment in the companion paper [166], where we scan the nuisance parameters using flat priors, and impose constraints on them within the likelihood function. We refer the reader to Sects. 2.1.2-2.1.4 and Sect. 3.1 of Ref. [166] for details. For ease of reference however, here we reproduce in Table 2 the parameter ranges and values used in our scans.

\subsection{Scanning methodology}

Our scanning methodology is similar to that detailed in Sect. 2.2 of the companion paper [166]; here we give only a short summary, focussing in particular on any points of difference.
We use a number of different priors, two different samplers, ${ }^{2}$ and a range of different sampler configurations to scan the parameter space of the MSSM7. We then combine the results of all scans into a single set of likelihood samples, and use it to generate profile likelihoods for the MSSM7 parameters and observables. Using multiple scanners, priors and sampling settings allows for accurate determination of both the highest-likelihood point and profile likelihood contours. We do not consider Bayesian posteriors in the current paper, as our preliminary investigations indicate that Bayesian results in weak-scale MSSM models are dominated by the choice of priors. This suggests that a careful choice of prior (based on e.g. fine-tuning considerations) is needed for later interpretation, which is beyond the scope of the current paper.

We primarily rely on the differential evolution scanner Diver 1.0.0 [169], but also perform some supplementary scans with the nested sampler MultiNest 3.10 [179]. For

\footnotetext{
${ }^{2}$ Here we use 'sampler' and 'scanner' synonymously.
} 
a performance comparison of Diver, MultiNest and other modern scanning algorithms, please see Sec. 11 of Ref. [169]. Unlike in the companion paper, we repeat all Diver scans with both self-adaptive jDE [180] and its $\lambda$ jDE variant [169]. The $\lambda \mathrm{jDE}$ algorithm is more aggressive in finding the maximum likelihood, whereas $j D E$ ensures a more thorough exploration of large regions of degenerate likelihood. Carrying out jDEonly scans (as opposed to retaining $\lambda \mathrm{jDE}$ for all scans, as in Ref. [166]) is more beneficial in the MSSM7 than in the NUHM2 or its subspaces, as the additional freedom of the MSSM7 means that individual regions satisfying the bound set by the DM relic density, whilst more numerous, occupy a more fragmented and isolated volume of the parameter space than in 'smaller' models like the CMSSM.

We carry out scans with effectively logarithmic priors using both Diver and MultiNest, where all parameters except $\tan \beta$ follow so-called "hybrid" priors. The hybrid prior we use for parameter $x$ of mass dimension $n$ is flat in the region $|x|<(100 \mathrm{GeV})^{n}$, and logarithmic for larger $|x|$. We supplement these scans with additional Diver runs using flat priors on the parameters most sparsely sampled at large values in log-prior scans $\left(A_{u_{3}}, A_{d_{3}}\right.$ and $\left.M_{H_{u}}^{2}\right)$, and additional MultiNest scans using flat priors on all dimensionful parameters. To further improve the completeness of the sampling across the parameter space, we also subdivide every run into separate scans for $M_{2}>0$ and $M_{2}<0$.

As we show in Sect. 4, chargino co-annihilation is the dominant mechanism for producing an acceptable DM abundance in large parts of the allowed parameter space. To ensure that we also explore the narrow parameter regions where sfermion co-annihilation or resonant annihilation is responsible for depleting the relic density to or below the observed value, we perform four 'directed' scans with different additional conditions on the particle spectrum:

1. squark co-annihilation - a cut on the mass of the lightest squark $m_{\tilde{q}_{1}}<1.5 m_{\tilde{\chi}_{1}^{0}}$,

2. slepton co-annihilation - a cut on the mass of the lightest slepton $m_{\tilde{l}_{1}}<1.75 m_{\tilde{\chi}_{1}^{0}}$,

3. heavy Higgs funnel - a cut on the mass of the CP-odd Higgs boson $1.8 m_{\tilde{\chi}_{1}^{0}}<m_{A}<2.2 m_{\tilde{\chi}_{1}^{0}}$, and

4. light Higgs funnel - a cut on the lightest neutralino mass $25<m_{\tilde{\chi}_{1}^{0}} / \mathrm{GeV}<85$.

For all four scans, we also require that $m_{\tilde{\chi}_{1}^{ \pm}}>1.05 m_{\tilde{\chi}_{1}^{0}}$, to avoid parameter combinations where chargino co-annihilation dominates. We carry out these scans exclusively with Diver, using pure jDE. In these directed scans, we allow $M_{2}$ to vary freely over its full positive and negative range (Table 1).

The mass conditions effectively act as priors, allowing us to obtain a starting population of points roughly in the right area, before refining the fit to the best-fit likelihood region corresponding to each mechanism for depleting the relic den- sity. Note that the bounds that we use for this process are quite generous compared to the actual definitions of these regions that we use later in this paper, as they are only designed to direct the scanner to the correct vicinity of parameter space in which to look for good fits, not to act as cut for physical interpretation. To generate an initial population of parameter points satisfying the cut in question, we simply draw randomly from the overall parameter space, and assign zero likelihood to all parameter combinations that do not satisfy the cut. We then take the set of resulting points satisfying the cut, and use them with Diver to discover new points with higher likelihood values, continuing until the algorithm indicates convergence (suggesting that the best fit has been found, to within some tolerance). The looser mass cut on sleptons compared to squarks ensures that we are able to generate an initial population within the required cut in a reasonable time.

Taking into account all sampling configurations, priors and parameter space partitionings, this leads to a total of 2 $\operatorname{sgn}\left(M_{2}\right) \times 2$ priors $\times(1$ MultiNest +2 Diver configurations $)$ +4 directed scans $=16$ scans. Each of the 16 scans took on the order of a few days to run on 2400 modern (Intel Core i7) cores. In total, these scans resulted in $1.8 \times 10^{8}$ valid samples, of which $1.76 \times 10^{7}\left(1.37 \times 10^{7}\right)$ were within the 2D 95\% (68\%) CL region of the global best-fit point. After the scans we ran all samples through a postprocessing step, using the postprocessor scanner of ScannerBit [169], in order to correct for a bug in the flavour likelihood and apply Run II LHC searches which had just recently become available in ColliderBit [181]. Following this postprocessing step $1.67 \times 10^{7}\left(2 \times 10^{5}\right)$ of the original samples ended within the $95 \%$ (68\%) CL region of the global best-fit point.

For the main Diver scans, we set the population size NP to 19200 , and the convergence threshold convthresh to $10^{-5}$; for the directed scans, we instead set $\mathrm{NP}=6000$ and convthresh $=10^{-4}$. For MultiNest, we use nlive $=5000$ live points and a stopping tolerance of $t \circ 1=0.1$. These are relatively loose choices, but this results from the fact that we are only using MultiNest to bulk out the dominant likelihood mode of each scan rather than to locate the best fit point with high accuracy (the later is performed by Diver).

\section{Observables and likelihoods}

We compute a wide range of collider, flavour, DM and precision observables, and combine them with results from the latest experimental searches to produce an extensive set of likelihood components, which we then use to construct a composite likelihood function for driving the samplers in this paper. The composite likelihood includes

- the DM relic density measurement $\Omega_{\mathrm{c}} h^{2}=0.1188 \pm$ 0.0010 from Planck [182] (implemented as an upper 
limit, so as to permit an additional non-neutralino DM component)

- Fermi-LAT Pass 8 dwarf limits on DM annihilation [183],

- IceCube 79-string limits on DM annihilation in the Sun [47, 184],

- direct DM searches by LUX [185-187], Panda-X [188], PICO [189, 190], XENON100 [191], SuperCDMS [192] and SIMPLE [193],

- the anomalous magnetic moment of the muon [194,195],

- MSSM contributions to the mass of the $W$ boson,

- 59 different flavour observables measured by $\mathrm{LHCb}$, Babar and Belle,

- 13 different ATLAS and CMS analyses from Run I and Run II (as in the companion paper [166], we apply the Run II searches as a postprocessing step),

- Higgs limits, mass measurements and signal strengths from LEP and the LHC, and

- limits from LEP on sparticle production and decay in 18 different channels.

- nuisance likelihoods associated the local density of DM [178], the quark contents of the nucleon [178], the top mass [196] and the strong coupling [196].

The theoretical treatments, experimental data and final likelihood functions for all these observables match those described in Sec. 3 of the companion paper [166], so we refer the reader to that paper, and the GAMBIT module papers $[178,181,196,197]$, for details. The only exception is the DM relic density calculation, which we perform for the MSSM7 with micrOMEGAs 3.6.9.2 [198] (with settings fast $=1$, Beps $=10^{-5}$ ), rather than DarkSUSY 5.1.3 [173], because the former is faster for some highly degenerate sfermion coannihilation models.

The observables that we include draw on many other external software packages: DDCalc 1.0.0 [178], FlexibleSUSY 1.5.1 ${ }^{3}$ [203], gamLike 1.0.0 [178], GM2Calc 1.3.0 [92], HiggsBounds 4.3.1 [204-206], HiggsSignals 1.4 [207], nulike 1.0.0 [47, 175], Pythia 8 8.212 [208], Superlso 3.6 [209-211] and SUSY-HIT 1.5 [212-215].

\section{Results}

\subsection{Best fits}

In much of the parameter space of the MSSM7 (and indeed the MSSM more generally), the annihilation cross-section of heavy neutralino DM is so small that the thermal relic

$\overline{3}$ FlexibleSUSY gets model-dependent information from SARAH $[199,200]$ and uses some numerical routines from SOFTSUSY [201, 202]. density greatly exceeds the value measured by Planck. Such models are robustly ruled out. The only way for a model to respect this upper limit is to exhibit one or more specific mechanisms for depleting the thermal abundance, typically associated with co-annihilation with another supersymmetric species, or resonant annihilation via a neutral boson 'funnel'.

Five such mechanisms play a role within the final $95 \%$ confidence level (CL) regions of our scans. In the following discussion, we classify samples and colour regions according which mechanism(s) they display:

- chargino co-annihilation: $\tilde{\chi}_{1}^{0} \geq 50 \%$ Higgsino,

- stop co-annihilation: $m_{\tilde{t}_{1}} \leq 1.2 m_{\tilde{\chi}_{1}^{0}}$,

- sbottom co-annihilation: $m_{\tilde{b}_{1}} \leq 1.2 m_{\tilde{\chi}_{1}^{0}}$,

- A/H funnel: $1.6 m_{\tilde{\chi}_{1}^{0}} \leq m_{\text {heavy }} \leq 2.4 m_{\tilde{\chi}_{1}^{0}}$,

$-h / Z$ funnel: $1.6 m_{\tilde{\chi}_{1}^{0}} \leq m_{\text {light }} \leq 2.4 m_{\tilde{\chi}_{1}^{0}}$,

where 'heavy' may be $H^{0}$ or $A^{0}$, and 'light' may be $h^{0}$ or $Z^{0}$, and a parameter combination qualifies as a member of a region if either condition is satisfied. Indeed, this is the strategy we adopt in general: if a model fulfils one of these conditions, we include it in the region, even if it ends up becoming a member of multiple regions, and even if some dominate over others. For clarity, we do not make any attempt to identify hybrid regions, or determine which of the mechanisms dominates (as to do so would require assumptions about relative temperature dependences and interferences between different partial annihilation rates). The union of these regions contain the full set of models allowed at 95\% CL.

In Table 3, we show the details of the best-fit point in each of these five regions, breaking down the final log-likelihood into contributions from the different observables included in the fit. The overall best fit occurs in the chargino coannihilation region, where the lightest two neutralinos and the lightest chargino are all dominantly Higgsino, and thus highly degenerate in mass. All pairwise annihilations and co-annihilations between any of these three species can thus contribute significantly to the final relic density in this region. In Fig. 1 we give a visual representation of the mass spectrum of this point, where one can see clearly that we have some very light neutralinos and charginos in this model. The masses are around $260 \mathrm{GeV}$, making them potentially interesting targets for future LHC searches (Sect. 5.1).

We also define a so-called 'ideal' reference likelihood in Table 3. This is the best likelihood that a model could realistically achieve were it to predict all observed quantities precisely, and predict no additional contribution beyond the expected background in searches that have produced only limits. We compute this for most likelihood components by assuming that the model prediction is either equal to the measured value or the background-only prediction. For some highly composite observables, where many different chan- 
Table 3 Best-fit points in the $A / H$-funnel, $\tilde{b}$ co-annihilation, $\tilde{t}$ coannihilation, $\tilde{\chi}_{1}^{ \pm}$co-annihilation and $Z / h$ funnel regions. For each point, we show the individual likelihood contributions, parameter values (including nuisance parameters) and derived quantities crucial for interpreting the mass spectrum. Other SM and astrophysical parameters are set to the fixed values given in Table 2. SLHA1 and SLHA2 files for the best-fit points can be found in the online data associated with this paper [170]

\begin{tabular}{|c|c|c|c|c|c|c|c|}
\hline Likelihood term & Ideal & $A / H$-funnel & $\tilde{b}$ co-ann. & $\tilde{t}$ co-ann. & $\tilde{\chi}_{1}^{ \pm}$co-ann. & $Z / h$-funnel & $\Delta \ln \mathcal{L}_{\mathrm{BF}}$ \\
\hline LHC sparticle searches & 0.000 & 0.000 & 0.000 & 0.000 & 0.000 & 0.000 & 0.000 \\
\hline LHC Higgs & -37.734 & -38.657 & -38.647 & -39.050 & -38.347 & -38.593 & 0.613 \\
\hline LEP Higgs & 0.000 & 0.000 & 0.000 & 0.000 & 0.000 & 0.000 & 0.000 \\
\hline ALEPH selectron & 0.000 & 0.000 & 0.000 & 0.000 & 0.000 & 0.000 & 0.000 \\
\hline ALEPH smuon & 0.000 & 0.000 & 0.000 & 0.000 & 0.000 & 0.000 & 0.000 \\
\hline ALEPH stau & 0.000 & 0.000 & 0.000 & 0.000 & 0.000 & 0.000 & 0.000 \\
\hline L3 selectron & 0.000 & 0.000 & 0.000 & 0.000 & 0.000 & 0.000 & 0.000 \\
\hline L3 smuon & 0.000 & 0.000 & 0.000 & 0.000 & 0.000 & 0.000 & 0.000 \\
\hline L3 stau & 0.000 & 0.000 & 0.000 & 0.000 & 0.000 & 0.000 & 0.000 \\
\hline L3 neutralino leptonic & 0.000 & 0.000 & 0.000 & 0.000 & 0.000 & 0.000 & 0.000 \\
\hline L3 chargino leptonic & 0.000 & 0.000 & 0.000 & 0.000 & 0.000 & 0.000 & 0.000 \\
\hline OPAL chargino hadronic & 0.000 & 0.000 & 0.000 & 0.000 & 0.000 & 0.000 & 0.000 \\
\hline OPAL chargino semi-leptonic & 0.000 & 0.000 & 0.000 & 0.000 & 0.000 & 0.000 & 0.000 \\
\hline OPAL chargino leptonic & 0.000 & 0.000 & 0.000 & 0.000 & 0.000 & 0.000 & 0.000 \\
\hline OPAL neutralino hadronic & 0.000 & 0.000 & 0.000 & 0.000 & 0.000 & 0.000 & 0.000 \\
\hline$B_{(s)} \rightarrow \mu^{+} \mu^{-}$ & 0.000 & -2.033 & -2.024 & -2.021 & -1.998 & -1.997 & 1.998 \\
\hline Tree-level B and D decays & 0.000 & -15.318 & -15.284 & -15.287 & -15.315 & -15.333 & 15.315 \\
\hline$B^{0} \rightarrow K^{* 0} \mu^{+} \mu^{-}$ & -184.260 & -194.316 & -195.283 & -193.103 & -194.734 & -195.551 & 10.474 \\
\hline$B \rightarrow X_{s} \gamma$ & 9.799 & 8.030 & 8.710 & 6.978 & 8.334 & 8.795 & 1.465 \\
\hline$a_{\mu}$ & 20.266 & 14.027 & 14.114 & 14.299 & 14.269 & 14.090 & 5.997 \\
\hline$W$ mass & 3.281 & 3.081 & 2.813 & 2.778 & 3.096 & 2.643 & 0.185 \\
\hline Relic density & 5.989 & 5.989 & 5.989 & 5.989 & 5.989 & 5.989 & 0.000 \\
\hline PICO-2L & -1 & -1.000 & -1.000 & -1.000 & -1.000 & -1.000 & 0.000 \\
\hline PICO-60 F & 0.000 & 0.000 & 0.000 & 0.000 & 0.000 & -0.001 & 0.000 \\
\hline SIMPLE 2014 & -2.972 & -2.972 & -2.972 & -2.972 & -2.972 & -2.972 & 0.000 \\
\hline LUX 2015 & -0.64 & -0.657 & -0.693 & -0.670 & -0.660 & -0.650 & 0.020 \\
\hline LUX 2016 & -1.467 & -1.501 & -1.574 & -1.527 & -1.506 & -1.487 & 0.039 \\
\hline PandaX 2016 & -1.886 & -1.909 & -1.960 & -1.927 & -1.912 & -1.899 & 0.026 \\
\hline SuperCDMS 2014 & -2.248 & -2.248 & -2.248 & -2.248 & -2.248 & -2.248 & 0.000 \\
\hline XENON100 2012 & -1.693 & -1.684 & -1.667 & -1.678 & -1.683 & -1.688 & 0.010 \\
\hline IceCube 79-string & 0.000 & -0.032 & 0.000 & 0.000 & -0.069 & 0.000 & 0.069 \\
\hline$\gamma$ rays (Fermi-LAT dwarfs) & -33.244 & -33.374 & -33.367 & -33.363 & -33.371 & -33.255 & 0.127 \\
\hline$\rho_{0}$ & 1.142 & 1.139 & 1.115 & 1.138 & 1.142 & 1.141 & 0.000 \\
\hline$\sigma_{s}$ and $\sigma_{l}$ & -6.115 & -6.115 & -6.117 & -6.115 & -6.128 & -6.116 & 0.013 \\
\hline$\alpha_{S}\left(m_{Z}\right)(\overline{M S})$ & 6.500 & 6.493 & 6.427 & 6.409 & 6.496 & 6.457 & 0.004 \\
\hline Top quark mass & -0.645 & -0.647 & -0.687 & -0.645 & -0.654 & -0.751 & 0.009 \\
\hline Total & -226.927 & -263.704 & -264.354 & -264.016 & -263.272 & -264.426 & 36.345 \\
\hline Quantity & & $A / H$-funnel & $\tilde{b}$ co-ann. & $\tilde{t}$ co-ann. & $\tilde{\chi}_{1}^{ \pm}$co-ann. & $Z / h$-funnel & \\
\hline$A_{d_{3}}(1 \mathrm{TeV})$ & & 9582.567 & 9669.750 & 9706.338 & 9376.461 & 1639.611 & \\
\hline$A_{u_{3}}(1 \mathrm{TeV})$ & & -9389.783 & 2957.229 & 2197.287 & 2923.877 & 3660.585 & \\
\hline$M_{2}(1 \mathrm{TeV})$ & & 3768.368 & 2404.020 & 1498.770 & 2469.296 & 2032.136 & \\
\hline $\tan \beta\left(m_{Z}\right)$ & & 7.133 & 11.862 & 12.743 & 46.632 & 19.058 & \\
\hline$m_{H_{u}}^{2}(1 \mathrm{TeV})$ & & $-1.271 \times 10^{7}$ & $-2.490 \times 10^{6}$ & $-9.757 \times 10^{5}$ & $-7.830 \times 10^{5}$ & $-6.077 \times 10^{5}$ & \\
\hline$m_{H_{d}}^{2}(1 \mathrm{TeV})$ & & $3.748 \times 10^{5}$ & $1.045 \times 10^{7}$ & $7.824 \times 10^{6}$ & $2.729 \times 10^{7}$ & $3.189 \times 10^{6}$ & \\
\hline
\end{tabular}


Table 3 continued

\begin{tabular}{|c|c|c|c|c|c|}
\hline Likelihood term & $A / H$-funnel & $\tilde{b}$ co-ann. & $\tilde{t}$ co-ann. & $\tilde{\chi}_{1}^{ \pm}$co-ann. & $Z / h$-funnel \\
\hline$m_{\tilde{f}}^{2}(1 \mathrm{TeV})$ & $9.680 \times 10^{7}$ & $9.229 \times 10^{6}$ & $3.006 \times 10^{6}$ & $1.352 \times 10^{7}$ & $9.574 \times 10^{6}$ \\
\hline$m_{t}$ & 173.289 & 173.120 & 173.325 & 173.445 & 172.990 \\
\hline$\alpha_{s}\left(m_{Z}\right)(\overline{M S})$ & 0.119 & 0.119 & 0.119 & 0.119 & 0.119 \\
\hline$\rho_{0}$ & 0.409 & 0.372 & 0.390 & 0.399 & 0.406 \\
\hline$\sigma_{s}$ & 42.966 & 43.242 & 42.916 & 44.101 & 42.591 \\
\hline$\sigma_{l}$ & 57.987 & 57.442 & 58.265 & 58.773 & 58.095 \\
\hline$M_{1}\left(M_{\mathrm{SUSY}}\right)$ & 2002.225 & 1242.861 & 767.869 & 1283.505 & 1053.133 \\
\hline$\mu\left(M_{\mathrm{SUSY}}\right)$ & 367.156 & 1477.923 & 987.697 & 253.479 & 69.449 \\
\hline$m_{\tilde{t}_{1}}$ & 9012.999 & 1237.689 & 759.551 & 2440.084 & 2132.455 \\
\hline$m_{\tilde{\tau}_{1}}$ & 9845.047 & 3034.359 & 1730.209 & 3698.869 & 3097.127 \\
\hline$m_{A}$ & 793.380 & 3567.851 & 2956.071 & 5348.470 & 1804.886 \\
\hline$m_{h}$ & 125.099 & 125.088 & 123.988 & 124.731 & 126.427 \\
\hline$m_{\tilde{\chi}_{1}^{0}}$ & 379.116 & 1233.050 & 759.524 & 258.939 & 69.247 \\
\hline (\%bino, \%Higgsino) & $(0,100)$ & $(98,2)$ & $(98,2)$ & $(0,100)$ & $(0,100)$ \\
\hline$m_{\tilde{\chi}_{2}^{0}}$ & -381.804 & -1491.708 & 994.456 & -262.754 & -73.665 \\
\hline (\%bino, \%Higgsino) & $(0,100)$ & $(0,100)$ & $(2,97)$ & $(0,100)$ & $(0,100)$ \\
\hline$m_{\tilde{\chi}_{1}^{ \pm}}$ & 380.734 & 1488.287 & 990.571 & 261.179 & 71.618 \\
\hline (\%wino, \%Higgsino) & $(0,100)$ & $(1,99)$ & $(2,98)$ & $(0,100)$ & $(0,100)$ \\
\hline$m_{\tilde{g}}$ & 12370.525 & 7920.520 & 5006.746 & 8104.365 & 6711.215 \\
\hline$\Omega h^{2}$ & $1.537 \times 10^{-2}$ & $3.890 \times 10^{-2}$ & $1.046 \times 10^{-2}$ & $8.027 \times 10^{-3}$ & $8.382 \times 10^{-4}$ \\
\hline
\end{tabular}

nels enter and the SM or background-only prediction can in principle be improved upon by introducing a BSM contribution, we take the ideal case to be the best fit achievable in a more general, effective phenomenological framework. The two likelihoods that we apply this treatment to are those associated with LHC measurements of Higgs properties, and the angular observables of the $B^{0} \rightarrow K^{* 0} \mu^{+} \mu^{-}$decay observed by $\mathrm{LHCb}$. In the former case, we take the ideal likelihood to be the best fit obtainable by independently varying the mass, width and branching fractions of a single Higgs in order to fit the LHC data contained in HiggsSignals. For the latter, we take the ideal likelihood from the best-fit point that we found in a flavour EFT global fit, discussed in Sec. 6.2 of the FlavBit paper [197].

The log-likelihood difference between the global best fit and the ideal case is $\Delta \ln \mathcal{L}_{\mathrm{BF}}=36.345$. Compared to the $\operatorname{CMSSM}\left(\Delta \ln \mathcal{L}_{\mathrm{BF}}=36.820 ; 4\right.$ BSM parameters +1 sign $)$, NUHM1 $\left(\Delta \ln \mathcal{L}_{\mathrm{BF}}=36.702 ; 5\right.$ BSM parameters +1 sign $)$ and NUHM2 $\left(\Delta \ln \mathcal{L}_{\mathrm{BF}}=36.362 ; 6\right.$ BSM parameters +1 sign) [166], we see a fairly mild improvement from moving to the MSSM7 (7 BSM parameters). It is possible to use $\Delta \ln \mathcal{L}_{\mathrm{BF}}$ to estimate the overall goodness of fit, but this requires knowledge of the effective number of degrees of freedom (see Sec. 4.1 of Ref. [166] for details). Guessing this to be between 30 and 50 (on the basis of the number

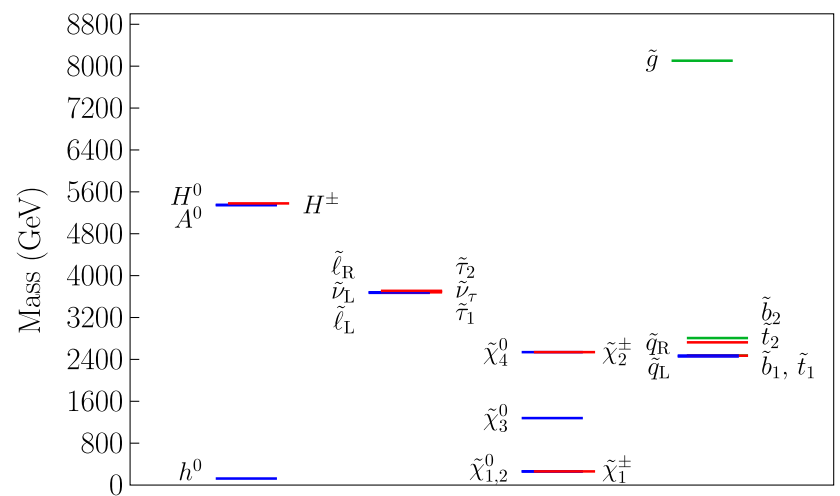

Fig. 1 Sparticle mass spectrum of the best-fit point

of active observables in the fit) would lead to a $p$ value of between $2 \times 10^{-5}$ and 0.02 . Comparing the specific case of e.g. 37 degrees of freedom to the equivalent calculation for the NUHM2, NUHM1 and CMSSM with 38, 39 and 40 degrees of freedom, respectively, the $p$ value for the MSSM7 would be $5.9 \times 10^{-4}$, compared to $5.9 \times 10^{-4}$ (NUHM2), $7.1 \times 10^{-4}$ (NUHM1) and $9.4 \times 10^{-4}$ (CMSSM). This comparison is not entirely fair, given that we have allowed $\operatorname{sgn}(\mu)$ to vary in the other three theories but not in the MSSM7. Nonetheless, it does suggest that the likelihood improve- 
ment in the MSSM7 is not sufficient to overcome the $p$ value penalty arising from the larger number of free parameters compared to the three GUT-scale models.

As with the CMSSM, NUHM1 and NUHM2, the individual contributions to $\Delta \ln \mathcal{L}_{\mathrm{BF}}=36.345$ come almost entirely from known anomalies unexplainable in either the SM or MSSM7. In particular, these include the $D$ and $D^{*}$ meson decay ratios $R_{D}$ and $R_{D^{*}}$ (contained in the tree-level $B$ and $D$ decay likelihood; see Sec. 5.1 of Ref. [197]), the magnetic moment of the muon ( $a_{\mu}$; see Sec. 4.2.2 of Ref. [196]) and the angular observables of the electroweak penguin decay $B^{0} \rightarrow K^{* 0} \mu^{+} \mu^{-}$(see Sec. 5.2 of Ref. [197]). We refer the reader to Sects. 4.1-4.3 of the companion paper [166] for further discussion.

The best fits possible by relic density mechanisms other than chargino co-annihilation are not drastically worse than the global best fit. The best models in the four other regions all lie within $\Delta \ln \mathcal{L}<1.2$ of the global optimum. Compared to the best fit with chargino co-annihilation, the best stop co-annihilation model has the light stop $\left(m_{\tilde{t}_{1}}=760 \mathrm{GeV}\right)$ needed to co-annihilate with the neutralino, and therefore a light sfermion spectrum more generally, due to the universality of $m_{\tilde{f}}$ at the weak scale. This point thus ends up being penalised by both the LHC Higgs likelihood and $B \rightarrow X_{s} \gamma$, but advantaged by $B^{0} \rightarrow K^{* 0} \mu^{+} \mu^{-}$. In contrast, the best-fit sbottom co-annihilation point has a heavier spectrum, with all sfermions masses above $1 \mathrm{TeV}$, and hence only suffers on $B^{0} \rightarrow K^{* 0} \mu^{+} \mu^{-}$relative to the global best fit. Both the light and heavy funnel best fits are hybrids with chargino co-annihilation, showing light charginos and neutralinos. The best-fit $A / H$ funnel point is only marginally worse than the global best, improving slightly on it in terms of $B^{0} \rightarrow K^{* 0} \mu^{+} \mu^{-}$but losing out due to slightly worse fits to $a_{\mu}, B \rightarrow X_{s} \gamma$ and the LHC Higgs likelihood. The spectrum of the best fit in the $Z / h$ funnel region is split, with heavy sfermions and gluinos, but light charginos and neutralinos. The latter lead to significant SUSY loop corrections to the $W$ self energy. This model is also slightly worse than the best fit in terms of $B^{0} \rightarrow K^{* 0} \mu^{+} \mu^{-}$and $a_{\mu}$, but recovers somewhat by making a smaller contribution to $B \rightarrow X_{s} \gamma$.

\subsection{Preferred regions}

We begin by giving the $1 \mathrm{D}$ profile likelihoods for each of our input parameters in Fig. 2. For simplicity, we refer to $m_{\tilde{f}} \equiv\left(m_{\tilde{f}}^{2}\right)^{1 / 2}$ rather than the input parameter $m_{\tilde{f}}^{2}$. We also give 1D profile likelihoods for the derived parameters $\mu$ and $M_{1}$. The GUT-inspired relation (Eq. 1) means that $M_{1} \approx$ $0.48 M_{2} \approx 0.18 M_{3}$, while $|\mu|$ is determined from the EWSB conditions. With $M_{1}<M_{2}$ it follows that $M_{1}$ and $\mu$ are the main mass parameters controlling the composition of the lightest neutralino. In our results we show $M_{1}$ and $\mu$ at the scale where the spectrum is calculated, $Q=\sqrt{m_{\tilde{t}_{1}} m_{\tilde{t}_{2}}} \equiv$ $M_{\text {SUSY }}$. Due to the central role played by the $\mu$ parameter, it is more instructive to discuss the results connected to the Higgs sector in terms of $\mu$ and $m_{A^{0}}$ than $m_{H_{u}}^{2}$ and $m_{H_{d}}^{2}$.

In Fig. 2 and throughout this paper, we show profile likelihood regions coloured according to the different coannihilation and funnel mechanisms contributing to keeping the neutralino relic density low enough to evade the Planck bound. These are: chargino co-annihilation (yellow), stop coannihilation (red), sbottom co-annihilation (blue), the $A / H$ funnel (orange) and the $h / Z$ funnel (purple); the definitions of these classifications can be found in the previous subsection.

Figure 3 shows the 2D joint profile likelihood for $M_{1}$ and $\mu$ (top) and $M_{2}$ and $m_{\tilde{f}}$ (bottom). The edges of the coloured regions here correspond to $95 \% \mathrm{CL}$ relative to the best fit of the entire sample, not relative to the best fits of each coloured region. Here we see that the parameter space allowed at $95 \%$ CL encompasses three distinct regions, each expressing a different composition for the lightest neutralino and chargino:

Region 1. $\mu<\left|M_{1}\right| . \tilde{\chi}_{1}^{0}$ and $\tilde{\chi}_{1}^{ \pm}$are mainly Higgsino. Region 2. $\mu \approx\left|M_{1}\right| \cdot \tilde{\chi}_{1}^{0}$ is a Higgsino/bino mixture and $\tilde{\chi}_{1}^{ \pm}$is dominantly Higgsino.

Region 3. $\mu>\left|M_{1}\right|$. $\tilde{\chi}_{1}^{0}$ is bino. As $\mu$ increases, $\tilde{\chi}_{1}^{ \pm}$ remains Higgsino-dominated up to $\mu \approx 2\left|M_{1}\right| \approx M_{2}$, after which the wino component dominates.

Due to Eq. 1, a purely wino-dominated $\tilde{\chi}_{1}^{0}$ is not possible in the MSSM7.

For Regions 1 and 2, the masses of the lightest chargino and the two lightest neutralinos are nearly degenerate, and all very close to $\mu$. The neutralino relic density is therefore depleted by all pairwise annihilations and co-annihilations between the three species, which we collectively refer to simply as 'chargino co-annihilation'. In Region 1, where the lightest neutralino is essentially a pure Higgsino, the relic density constraint implies $\mu \lesssim 1.2 \mathrm{TeV}$. The $A / H$-funnel also contributes across most of Regions 1 and 2, except in the case of very low $\mu$ or $\mu \ll\left|M_{1}\right|$, where the dependence of $m_{A^{0}}$ on $|\mu|$ makes it difficult to satisfy the funnel relation $m_{A^{0}} \sim 2 m_{\tilde{\chi}_{1}^{0}}$.

In Region 3, a small mass difference between the lightest neutralino and chargino is no longer automatic. The dominant relic density mechanisms in this parameter region are stop and sbottom co-annihilation, supported by annihilation through the $A$ / $H$ funnel. The tuning required in the former to get the lightest neutralino and lightest squark nearly degenerate in mass shows up as strongly-correlated bands in the $M_{2}-m_{\tilde{f}}$ plane (lower panels of Fig. 3). Because the MSSM7 employs a common sfermion soft-mass parameter $m_{\tilde{f}}^{2}$ at the input scale ( $Q=1 \mathrm{TeV}$ in our case), mass splittings among 

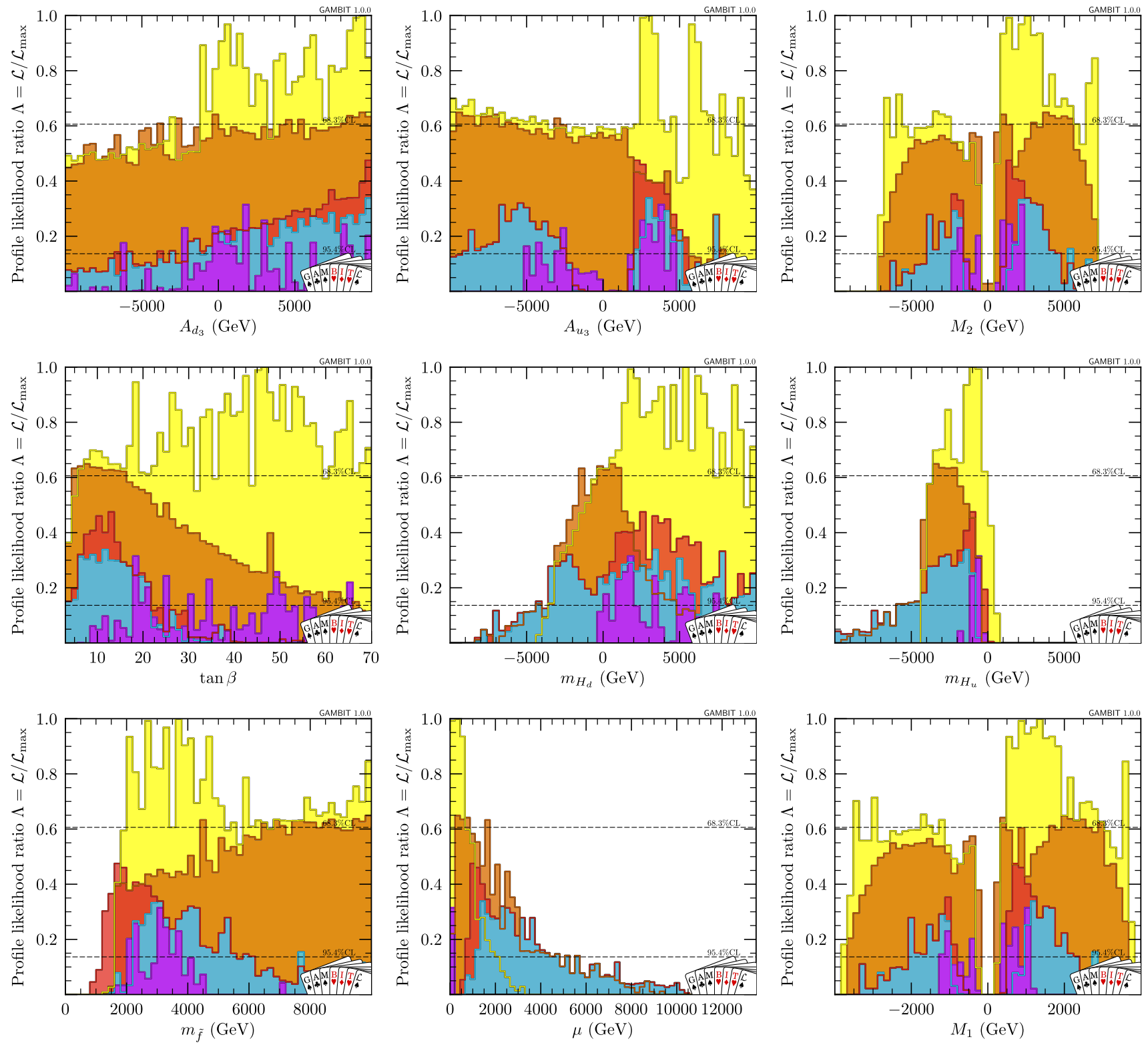

$\tilde{t}_{1}$ co-annihilation

$A / H$ funnel

$\tilde{\chi}_{1}^{ \pm}$co-annihilation

$\tilde{b}_{1}$ co-annihilation

$h / Z$ funnel

Fig. 2 1D profile likelihood ratio for the input parameters $A_{d_{3}}, A_{u_{3}}, M_{2}, \tan \beta, m_{H_{d}}, m_{H_{u}}$ and $m_{\tilde{f}}$, as well as the derived parameters $M_{1}$ and $\mu$

different sfermions are mostly generated by varying amounts of mixing. In comparison, the contribution from RGE running from $Q=1 \mathrm{TeV}$ to $Q=M_{\mathrm{SUSY}}$, which splits $m_{\tilde{f}}^{2}$ into individual soft masses, is generally subdominant.

In the tree-level stop mass matrix the off-diagonal element is $v y_{t}\left(A_{u_{3}} \sin \beta-\mu \cos \beta\right)$, while it is $v y_{b, \tau}\left(A_{d_{3}} \cos \beta-\right.$ $\mu \sin \beta$ ) in the sbottom and stau mass matrices, where $y_{t, b, \tau}$ are the fermion Yukawa couplings and $v \approx 246 \mathrm{GeV}$. Because increased left-right mixing reduces the mass of the lighter of the two mass eigenstates, the large top Yukawa ensures that $\tilde{t}_{1}$ is the lightest sfermion across most of the allowed parameter space (including for models that exhibit sbottom co-annihilation). With $3 \leq \tan \beta \leq 70$ the terms $A_{u_{3}} \sin \beta$ (stop) and $\mu \sin \beta$ (sbottom and stau) dominate the sfermion mixing in large regions of parameter space. The dependence on large $\mu$ to obtain a sbottom mass significantly lower than the mass set by the common $m_{\tilde{f}}$ parameter explains why the sbottom co-annihilation region does not extend as far to small $\mu$ as the stop co-annihilation region in Fig. 3. Also, since $y_{b} \approx 2.5 y_{\tau}$, the lightest stau remains heavier than the lightest sbottom in the regions of parameter space with large mixing for the down-type sfermions, which explains the absence of any region dominated by stau co-annihilation in our results. 
Fig. 3 Left: joint profile likelihoods in the $\mu-M_{1}$ (top) and $M_{2}-m_{\tilde{f}}$ planes (bottom). Stars indicate the point of highest likelihood in each plain, and white contours correspond to the $1 \sigma$ and $2 \sigma$ CL regions with respect to the best-fit point. Right: coloured regions indicating in which parts of the $2 \sigma$ best-fit region different co-annihilation and funnel mechanisms contribute to keeping the relic density low. The best-fit point in each region is indicated by a star with the corresponding colour
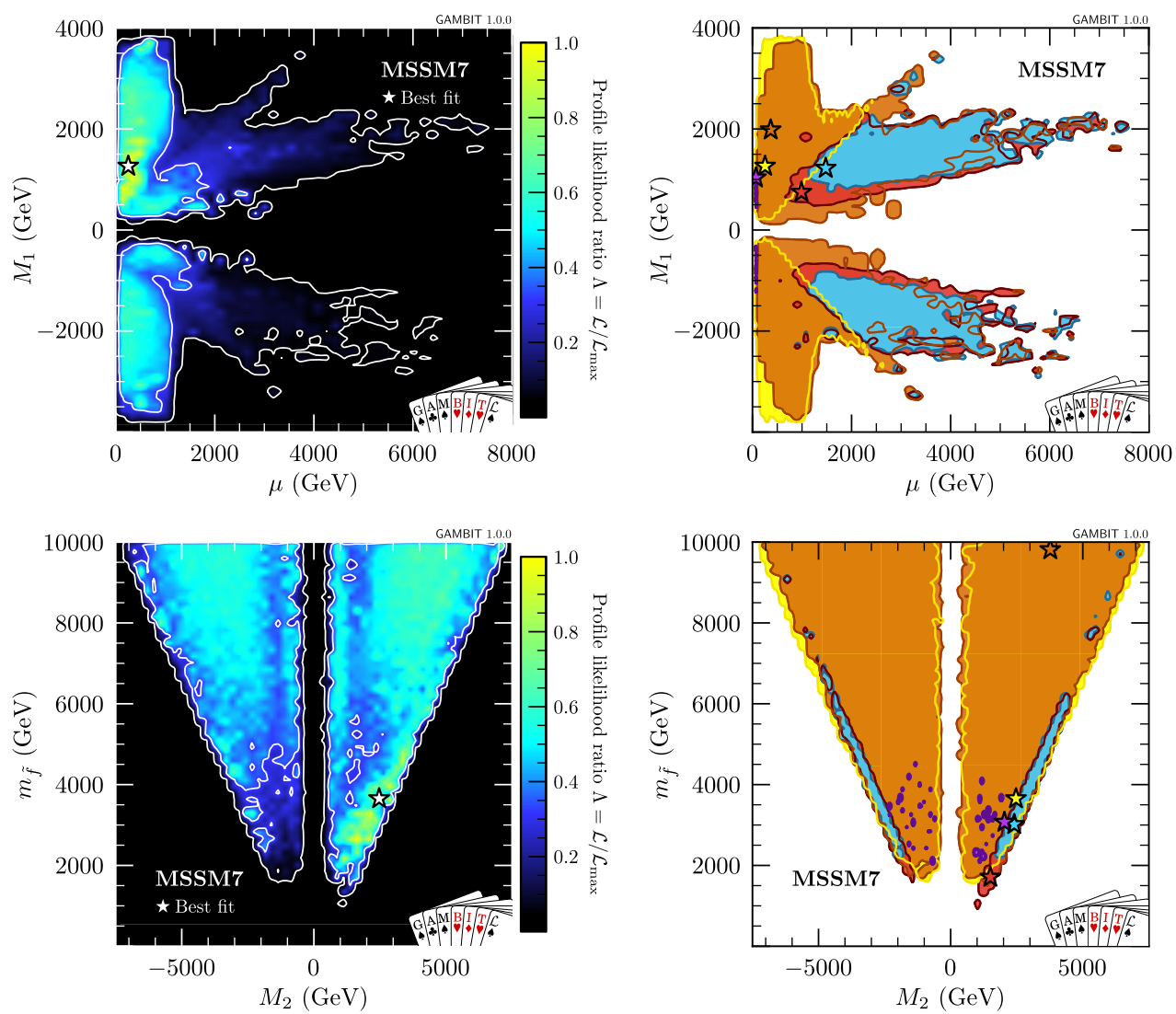

$\tilde{t}_{1}$ co-annihilation

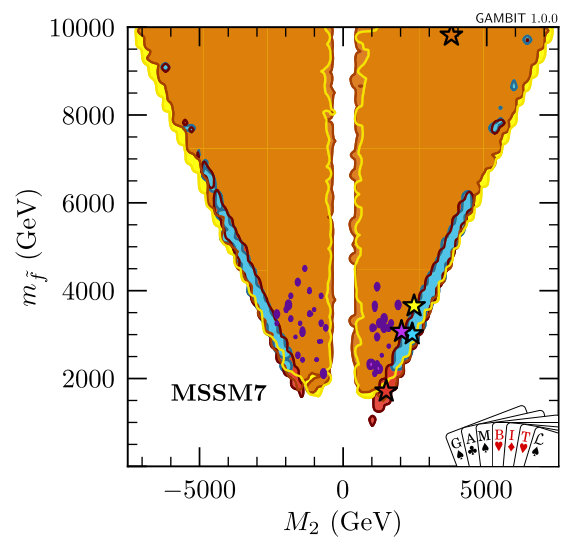

$\tilde{b}_{1}$ co-annihilation
The requirement that all sfermions are heavier than the lightest neutralino excludes large regions of parameter space at $m_{\tilde{f}} \lesssim 1.3\left|M_{2}\right| \approx 2.6\left|M_{1}\right|$ in the bottom panels of Fig. 3 . The steep slope of the exclusion boundary can broadly be understood as a consequence of the $\mu$-dependent mixing in the sfermion sector. The region close to the boundary at $m_{\tilde{f}} \approx 1.3\left|M_{2}\right|$ is part of Regions 2 and $3\left(\mu \gtrsim\left|M_{1}\right|\right)$ in the $\mu-M_{1}$ plane, so that increasing $M_{2}$ in this region pushes up both $M_{1}$ and $\mu$. To keep the lightest sfermion heavier than the neutralino, $m_{\tilde{f}}$ must therefore increase enough to compensate both the increase in neutralino mass from $M_{1}$ and the potential decrease in the light sfermion mass due to the $\mu$ dependent left-right mixing. We come back to this interplay between the parameters of the neutralino and sfermion sector when discussing the $\mu-\tan \beta$ plane in Fig. 5 .

The region of small $\left|M_{1}\right|$ in the upper row of Fig. 3 (and therefore also small $\left|M_{2}\right|$ in the lower row) is strongly constrained by LHC limits. Direct LHC searches are also strongly constraining at low $m_{\tilde{f}}$ (lower panels). Gluino searches are particularly effective, as Eq. (1) implies that the gluino mass parameter is $M_{3} \approx 7 M_{1}$ at a scale of $1 \mathrm{TeV}$. Given that simplified gluino mass limits reach up to $1.9 \mathrm{TeV}$ [216], this disfavours bino masses in the MSSM7 of up to $\sim 300 \mathrm{GeV}$. Indeed, this is the main reason that we do not find the same preference for very light binos observed in
Ref. [217], where each of the gaugino masses was allowed to vary independently. The common sfermion mass parameter means that, for light 3rd generation squarks, the 1st and 2nd generation squarks are not necessarily decoupled. Thus, LHC searches for 1st and 2nd generation squarks also constrain how far down towards low neutralino masses (small $\mu$ or $\left|M_{1}\right|$; upper panels of Fig. 3) and low sfermion masses (lower panels of Fig. 3) the stop and sbottom co-annihilation regions extend. Measurements of the $125 \mathrm{GeV}$ Higgs, limits from DM direct detection experiments, flavour physics and precision measurements of the $W$ mass also contribute to disfavouring low gaugino masses in our fits.

At low sfermion masses, we also see a weak preference for positive $M_{2}$, stemming from the $(g-2)_{\mu}$ likelihood. Because we assume $\mu>0$ for our model, having $M_{2}$ (and thus $M_{1}$ ) positive ensures a positive SUSY contribution to $(g-2)_{\mu}$.

In Fig. 4 we explore the impacts of the relic density constraint on the MSSM7 in more detail, investigating the profile likelihood of $\Omega h^{2}$ and $m_{A}$ as a function of the mass of the neutralino. The behaviour of Higgsino DM follows a relatively well-known pattern, seen also in the CMSSM and NUHM [166]: Higgsino DM co-annihilates steadily less efficiently as the neutralino mass increases, passing through the observed value of the relic density at $m_{\tilde{\chi}_{1}^{0}} \sim 1.2 \mathrm{TeV}$. At higher masses, exceeding the observed relic density can only be avoided 
Fig. 4 Left: joint profile likelihoods in the mass of the lightest neutralino and its relic density $\Omega h^{2}$ (top), and in the masses of the lightest neutralino and the CP-odd Higgs $A^{0}$ (bottom). Right: coloured regions indicating in which parts of the $2 \sigma$ best-fit region different co-annihilation and funnel mechanisms contribute to the relic density. The best-fit point in each region is indicated by a star with the corresponding colour
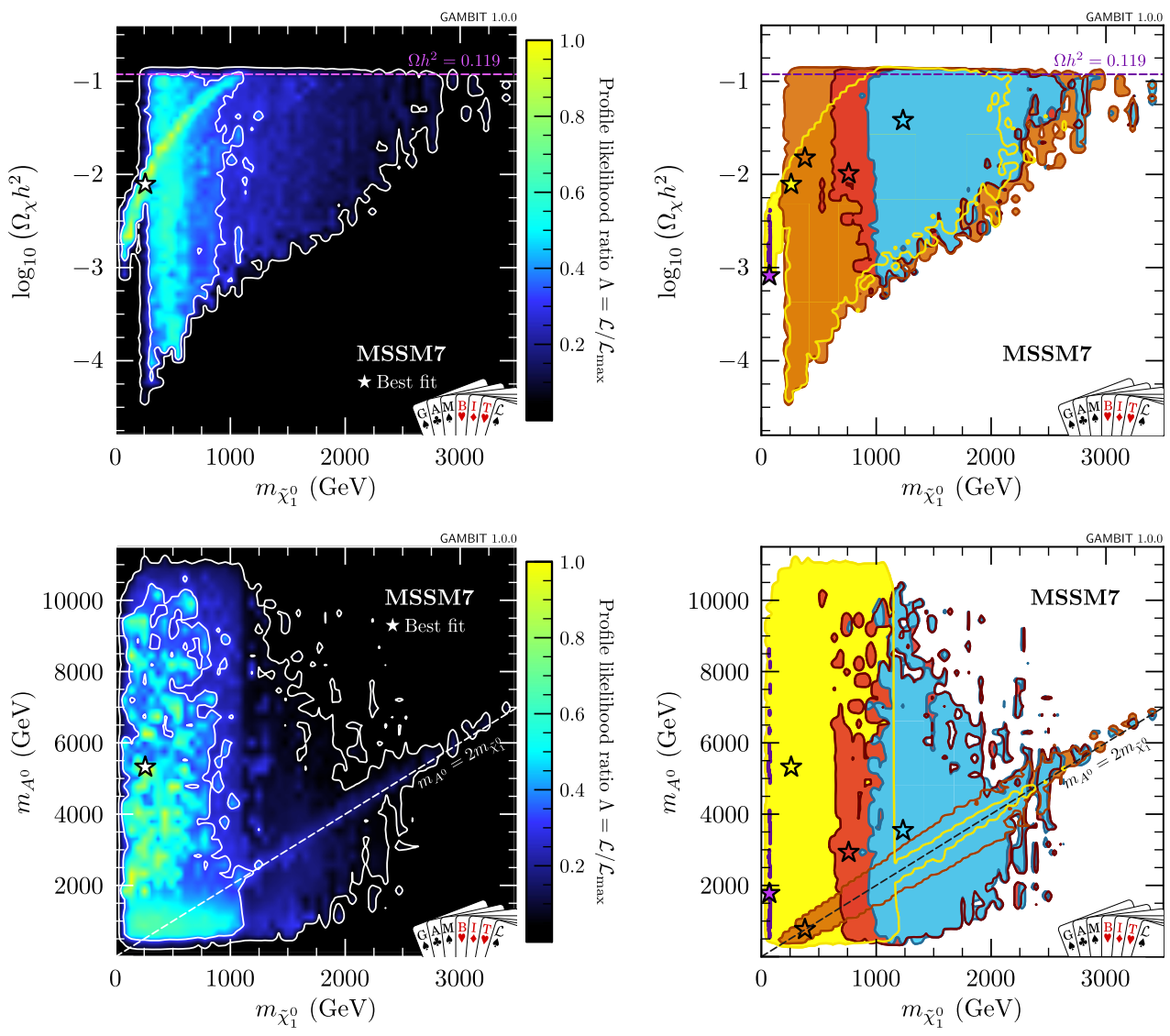

$\tilde{t}_{1}$ co-annihilation by resorting (whether in full or in part) to the heavy Higgs funnel or another co-annihilation mechanism - in this case, stop and/or sbottom co-annihilation. This can be seen in the lower-right panel of Fig. 4, where above $m_{\tilde{\chi}_{1}^{0}} \sim 1.2 \mathrm{TeV}$, the chargino co-annihilation region only exists along the funnel line $m_{A^{0}} \sim 2 m_{\tilde{\chi}_{1}^{0}}$.

At $m_{\tilde{\chi}_{1}^{0}} \lesssim 1.2 \mathrm{TeV}$, the efficiency of Higgsino coannihilation makes for sub-dominant Higgsino DM, as seen in the diagonal chargino co-annihilation region in the upperright panel of Fig. 4. This can be tempered by fine-tuning the Higgsino-bino mixture, bringing up the relic density to the observed value, but such combinations are now very strongly constrained by direct detection, where mixed gaugino-Higgsino DM maximises both the spin-dependent and spin-independent neutralino-nucleon scattering crosssections.

At very low masses, the chargino co-annihilation region reaches down far enough that resonant annihilation via the SM Higgs further boosts the annihilation cross-section, leading to a region of hybrid chargino co-annihilation- $h$ funnel models with neutralino masses as low as $61 \mathrm{GeV}^{4}$ The best

\footnotetext{
${ }^{4}$ For dedicated analyses of scenarios with a very light neutralino in MSSM parameterisations without a GUT relation on the gaugino masses, see for instance Refs. [218-222].
}

fit in this region (Table 3) has $m_{\tilde{\chi}_{1}^{0}}=69.2 \mathrm{GeV}, m_{\tilde{\chi}_{1}^{ \pm}}=$ $71.6 \mathrm{GeV}$ and $m_{\tilde{\chi}_{2}^{0}}=73.7 \mathrm{GeV}$, while the other sparticles are fairly heavy. This leads to considerable cross sections for direct pair production of $\tilde{\chi}_{1}^{0} \tilde{\chi}_{2}^{0}$ and $\tilde{\chi}_{1}^{+} \tilde{\chi}_{1}^{-}$at LEP. Indeed, such masses would naively seem to be in contradiction with published limits, e.g. $m_{\tilde{\chi}_{1}^{ \pm}}>94 \mathrm{GeV}[223,224]$. However, this particular limit assumes $m_{\tilde{\chi}_{1}^{ \pm}}-m_{\tilde{\chi}_{1}^{0}}>3 \mathrm{GeV}$, and does not strictly apply to our best fit. The GAMBIT implementation of LEP limits in ColliderBit, detailed in Sec. 2.2 of Ref. [181], takes into account the mass-dependent signal efficiency for the chargino and neutralino searches. These are quite important for cases where the spectrum has some degenerate masses, as in our best fit. In this case, the relevant search is the one for leptonic decays of the chargino at L3, with results shown in Fig. 2b of Ref. [225]. Our treatment is a significant improvement on the hard lower limits that have often been used in the past.

Figure 4 shows that the heavy Higgs funnel can work for a wide range of neutralino masses in the MSSM7, from $200 \mathrm{GeV}$ up to many $\mathrm{TeV}$. The lower limit here comes from the lower limit on the mass of the CP-odd Higgs boson, seen in the bottom-left corner of the $m_{A^{0}}-m_{\tilde{\chi}_{1}^{0}}$ plane (Fig. 4). This arises due to penalties from the flavour physics likelihoods and the LHC Higgs likelihood. Because $A^{0}$ is close 
Fig. 5 Left: joint profile likelihoods in $\mu$-tan $\beta$ (top) and $A_{u_{3}}-A_{d_{3}}$ planes. Right: coloured regions indicating in which parts of the $2 \sigma$ best-fit region different co-annihilation and funnel mechanisms contribute to the relic density. The best-fit point in each region is indicated by a star with the corresponding colour. In the bottom right plot the yellow chargino co-annihilation region covers the entire plane and the orange $A / H$ funnel region spans the entire plane below $A_{u 3} \sim 5 \mathrm{TeV}$
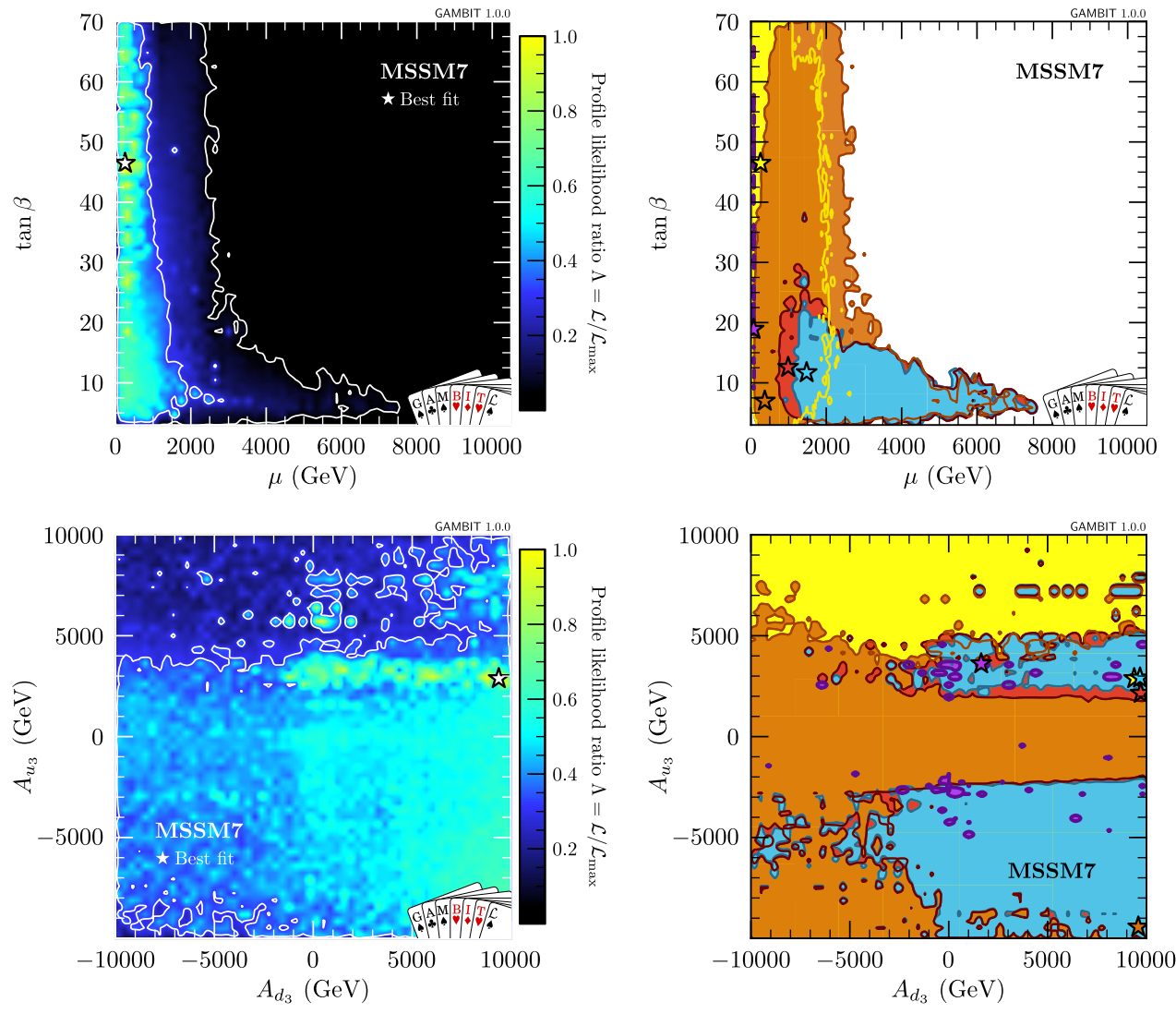

$\tilde{t}_{1}$ co-annihilation

$A / H$ funnel $\square \tilde{\chi}_{1}^{ \pm}$co-annihilation

$h / Z$ funnel in mass to $m_{H^{+}}\left(m_{H^{+}}^{2}=m_{A^{0}}^{2}+m_{W}^{2}\right.$ at tree level), having a light $A^{0}$ causes tension with the $B R\left(B \rightarrow X_{s} \gamma\right)$ likelihood, which in isolation requires $m_{H^{+}} \gtrsim 570 \mathrm{GeV}$ at $95 \%$ CL for type-II two Higgs doublet models such as the MSSM [226]. For large $\tan \beta$, the likelihoods for tree-level leptonic and semi-leptonic $B$ and $D$ decays also penalise low $A^{0}$ masses. The tension with these likelihoods at low masses is to some extent compensated for by an improvement in the fit to the electroweak penguin decay $B^{0} \rightarrow K^{* 0} \mu^{+} \mu^{-}$, but for $m_{A}^{0} \lesssim 400 \mathrm{GeV}$, the combined restrictions imposed by flavour physics and measurements of the $125 \mathrm{GeV} \mathrm{Higgs}$ push the likelihood below the 95\% CL, as evident in Fig. 4.

In this paper we have allowed neutralinos to be a subdominant component of DM. Were we to instead require that they constitute all of DM, our fits would be concentrated in the area around the horizontal line in the upper panels of Fig. 4. This would restrict the Higgsino-dominated DM models of the chargino co-annihilation region to $m_{\tilde{\chi}_{1}^{0}} \gtrsim 1 \mathrm{TeV}$, moving the best-fit point to the $A / H$ funnel and a mass of $m_{\tilde{\chi}_{1}^{0}}=416 \mathrm{GeV}$. In terms of the neutralino mass itself, this would rule out $m_{\tilde{\chi}_{1}^{0}}<250 \mathrm{GeV}$ at $95 \% \mathrm{CL}$ (1D). As we discuss later in this section, the absence of light charginos would also degrade the (already poor) fit to $a_{\mu}$.

In Fig. 5, we show the preferred regions and relic density mechanisms active in the $\mu-\tan \beta$ and $A_{d_{3}}-A_{u_{3}}$ planes.
The shape of the allowed region in the $\mu-\tan \beta$ plane can be understood as follows. For the scenario in Region 1 of the upper panels of Fig. 3, $\mu \ll M_{1}$ and the lightest neutralino is dominantly Higgsino. This leads to the relic density bound $\mu \lesssim 1.2 \mathrm{TeV}$. In Region 2 , where the lightest neutralino is a mixture of bino and Higgsino, this upper bound on $\mu$ increases to $\sim 2.5 \mathrm{TeV}$. This limit is where we see the edge of the chargino co-annihilation and $A / H$-funnel regions at intermediate and large $\tan \beta$ in the upper panels of Fig. 5.

In Region 3, $\mu>\left|M_{1}\right|$ and the lightest neutralino is dominantly bino, so there is no upper bound on $\mu$ from the relic density. In this case, the viable relic density mechanisms are stop/sbottom co-annihilation and the heavy Higgs funnel. Stop/sbottom co-annihilation can only work if the bino mass $\left(M_{1}\right)$ is similar to the mass of the lightest squark. At large $\tan \beta$, the left-right mixing in the sbottom mass matrix is proportional to $\mu$, meaning that to keep the sbottom from becoming tachyonic, the diagonal entry $\left(m_{\tilde{f}}^{2}\right)$ must be increased as $\mu$ is increased. Pulling up $m_{\tilde{f}}^{2}$ therefore pulls up the mass of the lightest squark, which in turn requires pulling $\left|M_{1}\right|$ up in order to stay in the stop/sbottom co-annihilation region. This is a delicate game, as $\left|M_{1}\right|$ needs to be kept below $\mu$ in order to remain in the bino LSP region (Region 3) at all. Whether or not this is possible depends on small corrections 
from other parameters. At smaller values of $\tan \beta$, the leftright mixing picks up an additional contribution proportional to $A_{d_{3}}$, and the adjustment can be pulled off with the help of some additional tuning in $A_{d_{3}}$. The net result is that $\left|M_{1}\right|$ remains less than $\mu$, but not by more than a factor of a few. Because the heavy Higgs bosons receive their dominant mass contribution from $|\mu|$, this sets their masses to be a factor of a few times that of the lightest neutralino, making stop/sbottom co-annihilation at higher $\mu$ in Region 3 appear mostly as a hybrid with the $A / H$ funnel.

At large $\mu$ and large $\tan \beta$, models in Region 3 are also impacted significantly by the Higgs likelihood. As discussed in Refs. [227,228], the bottom Yukawa coupling receives important SUSY corrections proportional to $\mu \tan \beta$, coming from gluino-sbottom and charged Higgsino-stop loops. For large $\mu$ and $\tan \beta$, this increases the decay rate $\Gamma\left(h^{0} \rightarrow \bar{b} b\right)$, which reduces the signal strengths for all other Higgs channels. The gluino-sbottom contribution is generally dominant, and for $\mu>0$ it is always positive. On the other hand, the Higgsino-stop contribution is proportional to $A_{u_{3}}$, so that for large and negative $A_{u_{3}}$ it can compensate the gluino-sbottom correction. Thus, the good-fit region extending out towards large $\mu$ is dominantly associated with large, negative $A_{u_{3}}$.

Large $\left|A_{u_{3}}\right|$ may cause the scalar potential of the MSSM to develop a minimum that breaks gauge invariance. We checked this in the same way as described in Sec. 4.1 of the companion paper [166], finding even less impact in the MSSM7 than in the CMSSM or NUHM: whilst a small number of individual points are potentially affected by colour- or charge-breaking vacua, the overall preferred regions of the model remain unaffected. We naively carried out the same tests for $\left|A_{d_{3}}\right|$ as well, swapping all up-type parameters for their down-type equivalents. We found that a few more models were affected than in the up-type tests, in particular those at large $\mu$ and small $\tan \beta$ discussed in the context of Fig. 5 above, where $A_{d_{3}}$ helps to prevent the sbottoms becoming tachyonic. However, the impact was still quite isolated and had no impact on the overall inference.

In Fig. 6, we show the profile likelihood for the SUSY contribution $\Delta a_{\mu}$ to the magnetic moment of the muon, compared with the experimental likelihood function for the observed discrepancy $a_{\mu, \mathrm{obs}}-a_{\mu, \mathrm{SM}}=(28.7 \pm 8.0) \times 10^{-10}$. Chargino co-annihilation models give the largest SUSY contributions, as they exhibit lighter charginos than other models. However, due to the relatively large values preferred for $m_{\tilde{f}}$, which governs the masses of $\tilde{\mu}$ and $\tilde{v}_{\mu}$, it is essentially impossible to fit $a_{\mu}$ simultaneously with all other observables even in the chargino co-annihilation region.

Compared to the MSSM10 results discussed in Ref. [217], we see broadly similar and consistent phenomenology, up to differences expected from the slightly different models being scanned. Both studies find the light Higgs funnel, chargino co-annihilation and stop/sbottom co-annihilation in essen-

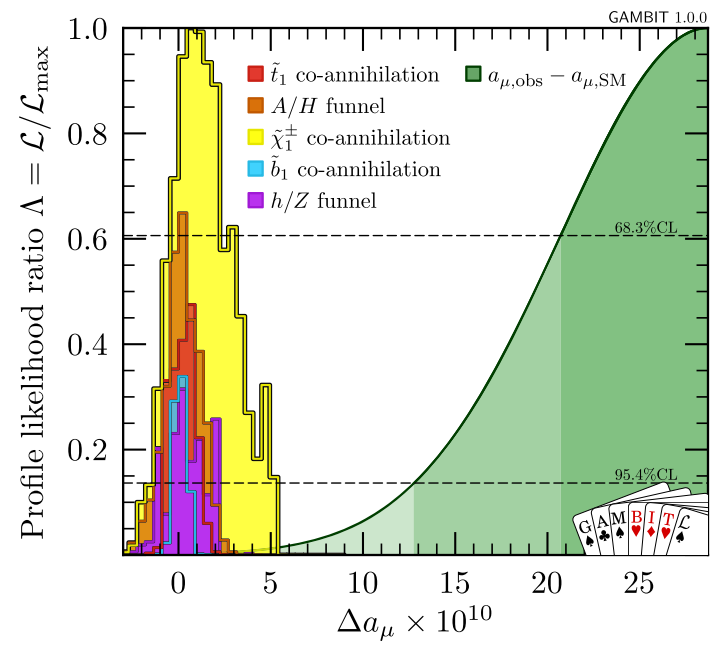

Fig. 6 1D profile likelihood ratio for the SUSY contribution $\Delta a_{\mu}$ to the anomalous magnetic moment of the muon. In green we show a Gaussian likelihood for the observed value $a_{\mu, \mathrm{obs}}-a_{\mu, \mathrm{SM}}=(28.7 \pm 8.0) \times 10^{-10}$, where we have combined the experimental and Standard Model (SM) theoretical uncertainties in quadrature

tially the same areas. As already discussed, we find that the MSSM7 does not permit stau co-annihilation, and we see a preference for larger neutralino and sfermion masses than Ref. [217], a consequence of the unified gaugino and sfermion mass parameters in the MSSM7 and our inclusion of constraints from Run II of the LHC. We also see stop/sbottom co-annihilation extend to higher masses than in Ref. [217], reflecting either a lower likelihood for such models relative to the best fit in the MSSM10 than in the MSSM7, or improved sampling in the current paper. Unlike in the MSSM10, we find that it is not possible to consistently explain $a_{\mu}$ in the MSSM7.

\section{Future prospects}

\subsection{LHC}

In Fig. 7 we show the 1D profile likelihoods for the masses of $\tilde{\chi}_{1}^{0}, \tilde{\chi}_{1}^{ \pm}, \tilde{g}, \tilde{t}_{1}, \tilde{b}_{1}$ and $\tilde{\tau}_{1}$. The $2 \sigma$ preferred region for the gluino mass extends upwards from $\sim 2 \mathrm{TeV}$, which is on the border of exclusion by current LHC searches for 0-lepton final states, to $\sim 20 \mathrm{TeV}$, well beyond the reach of the LHC. Similarly for $m_{\tilde{\tau}_{1}}$, where the small, weak production crosssection ensures that the predicted mass range is currently unobservable at the LHC.

More interesting are the $\tilde{\chi}_{1}^{0}$ and $\tilde{\chi}_{1}^{ \pm}$profile likelihoods, which are both peaked at low values. Given that these are naively within range of both LEP and the LHC Run I analyses, it is worth examining the properties of these low mass points in detail. Figure 8 shows our profile likelihood function in the $\tilde{\chi}_{1}^{ \pm}-\tilde{\chi}_{1}^{0}$ mass plane, zoomed into the low-mass region, 

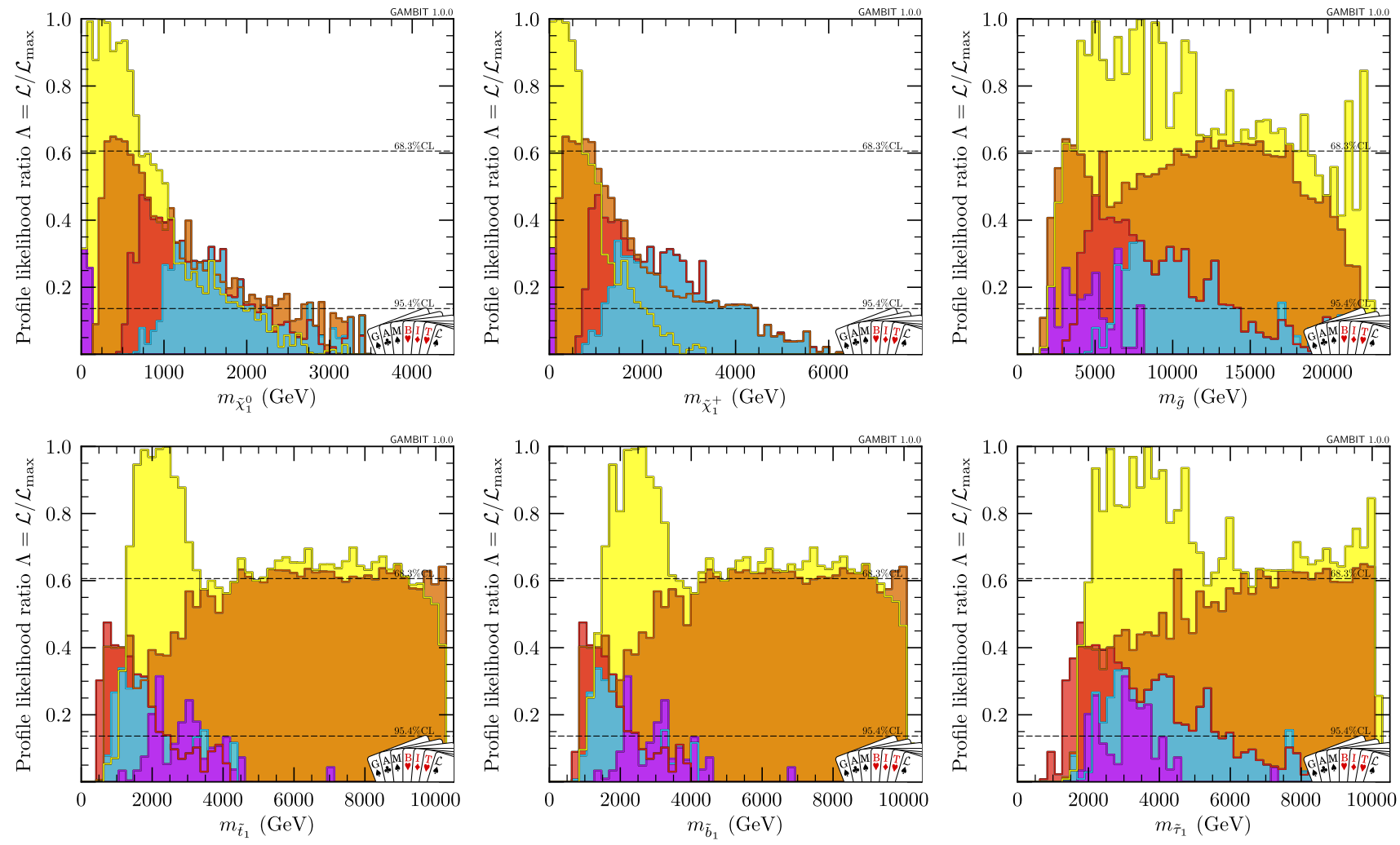

$\tilde{t}_{1}$ co-annihilation

$A / H$ funnel $\square \tilde{\chi}_{1}^{ \pm}$co-annihilation

$\tilde{b}_{1}$ co-annihilation

$h / Z$ funnel

Fig. 7 1D profile likelihood ratios for the masses $m_{\tilde{\chi}_{1}^{0}}, m_{\tilde{\chi}_{1}^{+}}, m_{\tilde{g}}, m_{\tilde{t}_{1}}, m_{\tilde{b}_{1}}$ and $m_{\tilde{\tau}_{1}}$. We show separate distributions for each mechanism that allows the models to obey the relic density constraint

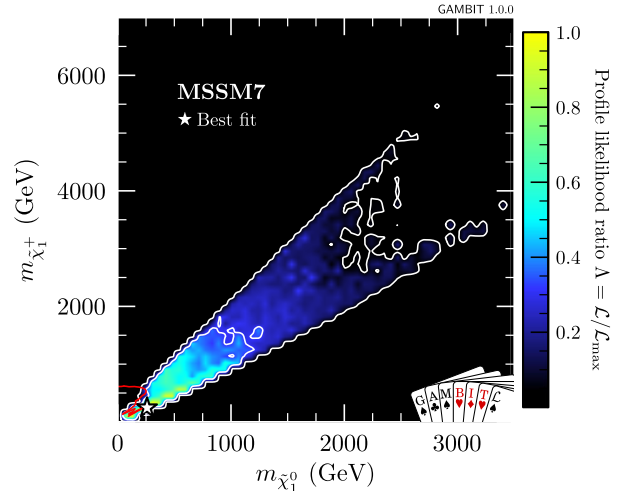

$\tilde{t}_{1}$ co-annihilation

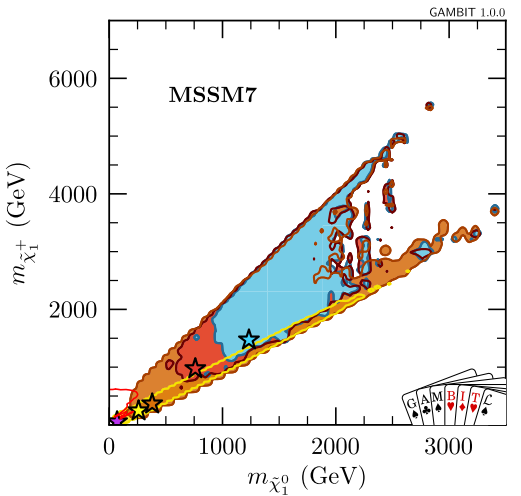

$\tilde{\chi}_{1}^{ \pm}$co-annihilation

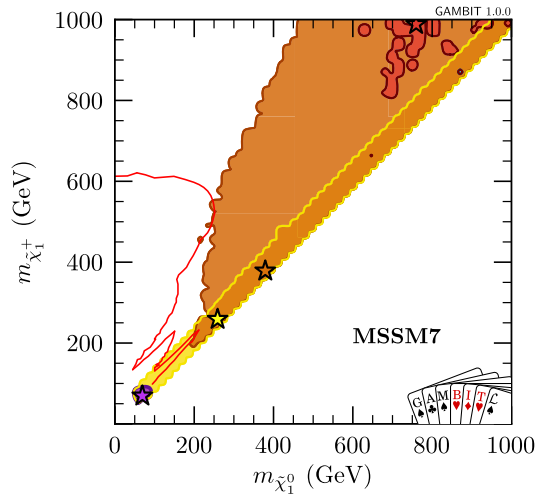

$\tilde{b}_{1}$ co-annihilation

$h / Z$ funnel
Fig. 8 Left: profile likelihood in the $\tilde{\chi}_{1}^{ \pm}-\tilde{\chi}_{1}^{0}$ mass plane. Centre: subregions within the $95 \%$ CL profile likelihood region, coloured according to mechanisms by which the relic density constraint is satisfied. The regions shown correspond to neutralino co-annihilation with charginos, stops or sbottoms, and resonant annihilation through the light or heavy Higgs funnels. Superimposed in red is the latest CMS Run II simplified model limit for $\tilde{\chi}_{1}^{ \pm} \tilde{\chi}_{1}^{0}$ production and decay with decoupled slep- tons [229]. This limit should be interpreted with caution (see main text for details). Right: the same information as the central plot, but zoomed into the low-mass region. Note that, although the CMS limit appears to have excluded part of the chargino co-annihilation region, this is a binning effect. One should instead refer to the plot of the $\tilde{\chi}_{1}^{ \pm}-\tilde{\chi}_{1}^{0}$ mass difference in Fig. 7, which provides finer resolution on the mass difference in this region along with colour-coding indicating which mechanisms help to satisfy the relic density constraint. For the part of our $2 \sigma$ region with $m_{\tilde{\chi}_{1}^{ \pm}} \lesssim 275 \mathrm{GeV}$, an acceptable relic density is mostly generated via chargino co-annihilation, leading to very degenerate $\tilde{\chi}_{1}^{ \pm}$and $\tilde{\chi}_{1}^{0}$ masses. This explains the lack of exclusion by the LEP and LHC analyses included in our scan 

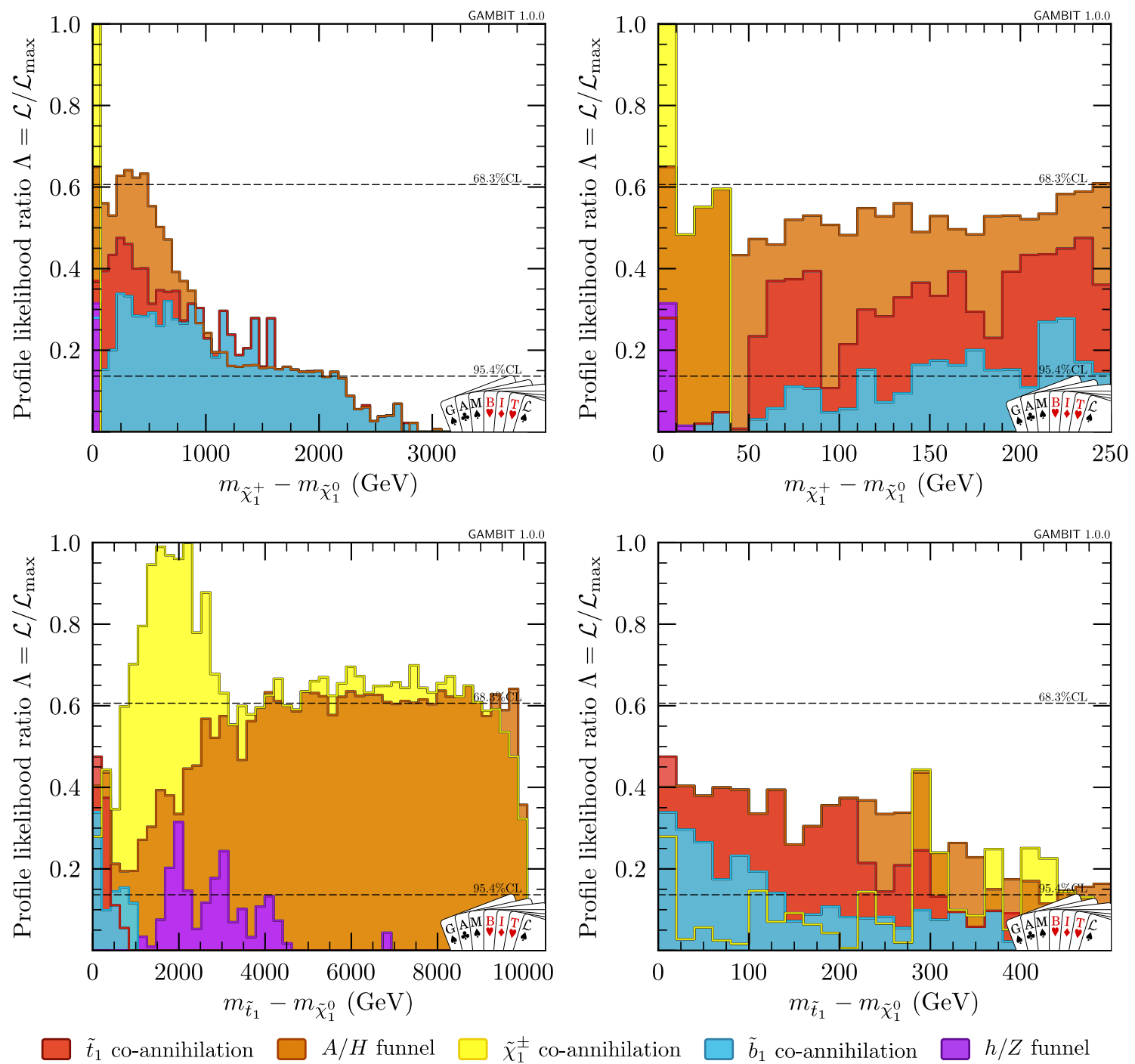

Fig. $91 \mathrm{D}$ profile likelihood ratios for the $\tilde{\chi}_{1}^{ \pm}-\tilde{\chi}_{1}^{0}$ mass difference (top) and the $\tilde{t}_{1}-\tilde{\chi}_{1}^{0}$ mass difference (bottom). Left: separate distributions for each mechanism allowing models to obey the relic density constraint. The regions correspond to neutralino co-annihilation with

charginos, stops or sbottoms, and resonant annihilation through the light or heavy Higgs funnels. Right: as per the left, but zoomed in to small mass differences

(which lose sensitivity for compressed spectra). Notably, our more careful treatment of the LEP limits than in previous studies has allowed models within the naive LEP reach to emerge unscathed.

One might wonder if other LHC analyses will soon (or have already) probed this low-mass region. The most recent EW gaugino limits are from CMS [231-234], using $36 \mathrm{fb}^{-1}$ of $13 \mathrm{TeV}$ data. A detailed study of the impact of these results would require the addition of the relevant analyses to the ColliderBit module, and the calculation of a complete likelihood similar to the equivalent Run I analyses already included in ColliderBit. In the present case, however, we can already obtain some insight from a more basic analysis of the simplified model limits presented by the CMS Collaboration. CMS interpreted their results for each final state in a range of

simplified models of chargino and neutralino production, in which they set the branching fractions for specific decays to $100 \%$, fixed the gaugino content, and set a 95\% CL exclusion limit in the $\tilde{\chi}_{1}^{ \pm}-\tilde{\chi}_{1}^{0}$ mass plane. Figure 7 demonstrates that the sleptons are heavy across our entire preferred $2 \sigma$ region, which is a natural consequence of having a unified sfermion mass in our parameterisation of the MSSM7. At least one stop mass must be high to induce large radiative corrections to the Higgs mass, which has the effect of dragging up the sfermion mass scale. In addition, and as mentioned previously, the $\tilde{\tau}_{1}$ will typically be heavier than the $\tilde{t}_{1}$ and $\tilde{b}_{1}$ due to the smaller Yukawa coupling. Thus, the relevant CMS simplified models are those featuring decoupled sleptons [229]. We caution that these limits do not apply in general, and do not directly translate to limits on our model points without a detailed check 


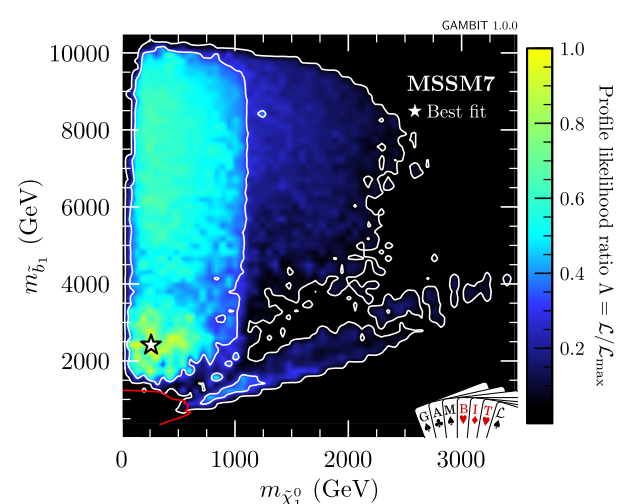

$\tilde{t}_{1}$ co-annihilation

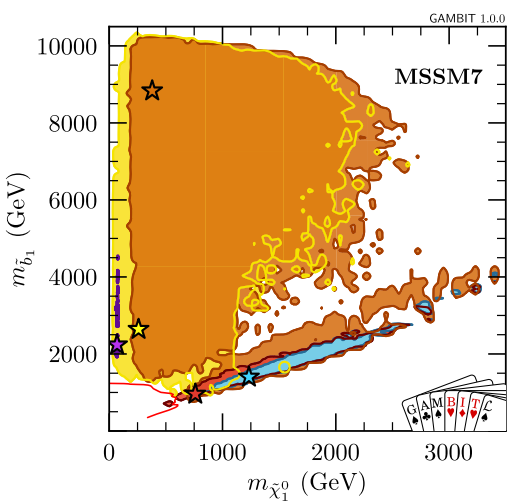

$\tilde{\chi}_{1}^{ \pm}$co-annihilation

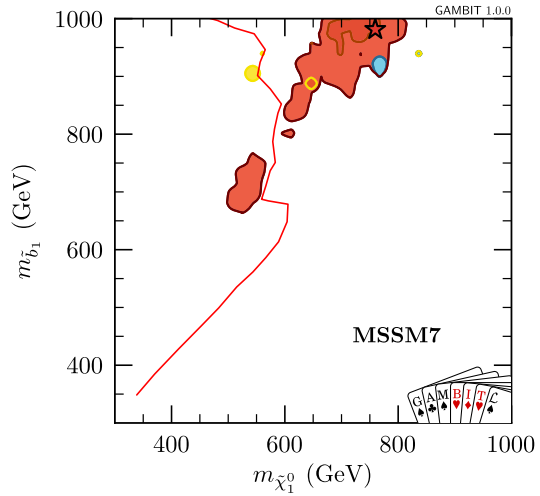

$\tilde{b}_{1}$ co-annihilation

$h / Z$ funnel
Fig. 10 Left: The profile likelihood ratio in the $\tilde{b}_{1}-\tilde{\chi}_{1}^{0}$ mass plane. Centre: Colour-coding shows mechanism(s) that allow models within the $95 \%$ CL region to avoid exceeding the observed relic density of DM. The regions shown correspond to neutralino co-annihilation with charginos, stops or sbottoms, and resonant annihilation through the light or heavy Higgs funnels. Right: The same information as the central plot, zoomed into the low-mass region that the neutralino and chargino mixing matrices and decay branching fractions match the CMS assumptions. One can, however, treat the CMS limits as the most optimistic possible exclusion in the $\tilde{\chi}_{1}^{ \pm}-\tilde{\chi}_{1}^{0}$ plane, to obtain a rough guide to the CMS sensitivity.

Proceeding in this spirit, we see that the current CMS limits just barely touch our $2 \sigma$ contour in regions where the spectrum is not compressed (Fig. 8). Indeed, the highest likelihood region looks to be out of reach in the near future. Note that if the GUT-inspired constraint on $M_{2}$ is relaxed, more solutions would fall within the CMS exclusion limit, so these searches will be important for global fits with more parameters. For compressed spectra, the details are less clear, as the ability of the CMS soft dilepton search to exclude the lightest models depends crucially on the precise $\tilde{\chi}_{1}^{ \pm}-\tilde{\chi}_{1}^{0}$ mass splitting. This is shown in the top of Fig. 9, where it is apparent that the chargino co-anihilation points appear as a peak in the likelihood at $\tilde{\chi}_{1}^{ \pm}-\tilde{\chi}_{1}^{0}$ mass differences of less than $10 \mathrm{GeV}$. This is too small to be probed by the recent CMS results. The chargino co-annihilation region remains free from LHC exclusion, assuming prompt decays of the chargino. We note, however, that for very small mass differences (approaching the pion mass), long-lived particle searches might provide additional constraints. We defer a detailed analysis of these to future work.

We now look at whether it is possible to probe the squark sector of the MSSM7 at the LHC in the near future. The lightest squarks are the $\tilde{t}_{1}$ and $\tilde{b}_{1}$. Figure 7 shows that the peak of the sbottom profile likelihood lies out of reach of the LHC in the near future, and that masses below $\sim 800 \mathrm{GeV}$ are disfavoured at the $2 \sigma$ level. Figure 10 shows the $\tilde{b}_{1}-\tilde{\chi}_{1}^{0}$ mass plane, revealing that the lower sbottom masses are associated with a small $\tilde{b}_{1}-\tilde{\chi}_{1}^{0}$ mass difference. This arises from the fact that stop and/or sbottom co-annihilation often account for the generation of an acceptable relic density in this low-mass region. However, there are also low-mass regions in which resonant $A / H$ annihilation or chargino co-annihilation contribute to DM annihilation, giving a wider range of mass differences. As above, comparison with recent CMS simplified model limits provides some insights into the ability of the LHC to probe these models in the near future. A variety of CMS searches for sbottom production have been interpreted in the context of a simplified model of sbottom pair production and decay to a bottom quark and the lightest neutralino [235-237]. We again treat these limits as a rough guide to the most favourable possible LHC exclusion potential, and compare our results to the CMS summary plot given in Reference [238]. The current analyses have potentially probed a small region of Fig. 10 (with $\tilde{\chi}_{1}^{0}$ masses below $600 \mathrm{GeV}$ and $\tilde{b_{1}}$ masses below $\approx 1 \mathrm{TeV}$ ). However, almost our entire $2 \sigma$ preferred region remains unconstrained. Directly ruling out sbottom co-annihilation as a viable contributor to an acceptable relic density would require probing compressed spectra in sbottom decays up to a mass of $\sim 4 \mathrm{TeV}$, an impossible task at the LHC. Nonetheless, the fact that current limits are nearing the tip of the stop co-annihilation strip means that discovery prospects even in the next run of the LHC are quite promising (although more so for models that exhibit only stop co-annihilation than those that display both stop and sbottom co-annihilation).

The stop mass has a marginally higher likelihood at lower masses (Fig. 7). Figure 11 shows the profile likelihood ratio in the $\tilde{t}_{1}-\tilde{\chi}_{1}^{0}$ mass plane, along with colour-coded regions illustrating the relevant relic density mechanisms. As for the sbottom mass, points with a $\tilde{t}_{1}$ mass below $1 \mathrm{TeV}$ show a strong mass correlation with the lightest neutralino, as they lie in the stop co-annihilation region. Comparison with the most recent CMS Run II simplified model results [236,237,242-244] 


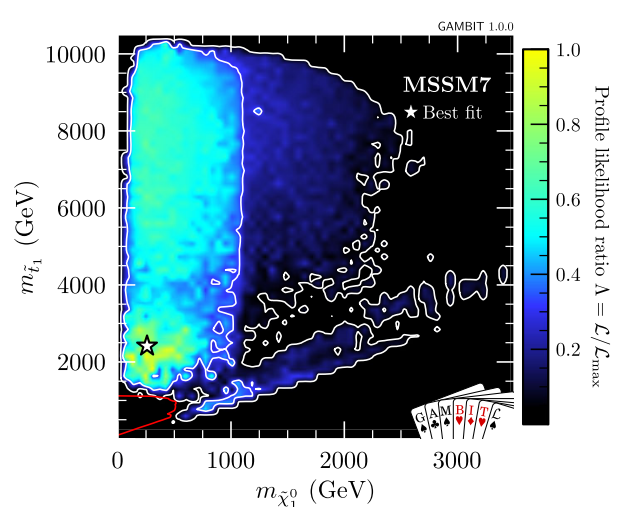

$\tilde{t}_{1}$ co-annihilation
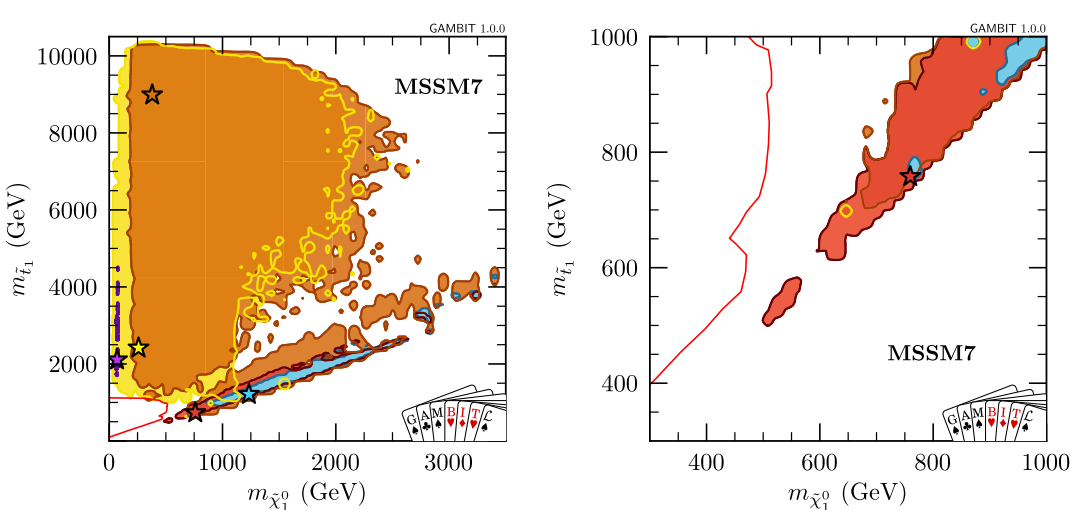

$\tilde{\chi}_{1}^{ \pm}$co-annihilation

$\tilde{b}_{1}$ co-annihilation

$h / Z$ funnel
Fig. 11 Left: the profile likelihood ratio in the $\tilde{t}_{1}-\tilde{\chi}_{1}^{0}$ mass plane. Centre: colour-coding shows mechanism(s) that allow models within the $95 \% \mathrm{CL}$ region to avoid exceeding the observed relic density of DM. The regions shown correspond to neutralino co-annihilation with charginos, stops or sbottoms, and resonant annihilation through the light or heavy Higgs funnels. Superimposed in red is the latest CMS Run II simplified model limit for stop pair production [230]. Right: the same information as the central plot, zoomed into the low-mass region

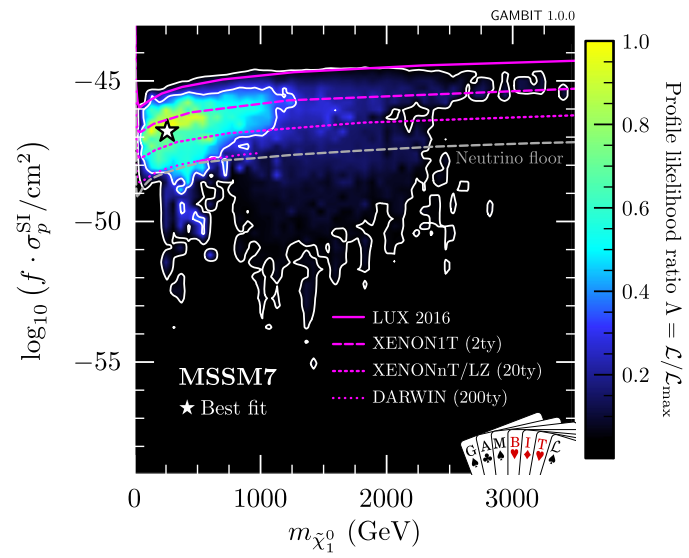

$\tilde{t}_{1}$ co-annihilation

$A / H$ funnel

$\tilde{\chi}_{1}^{ \pm}$co-annihilation

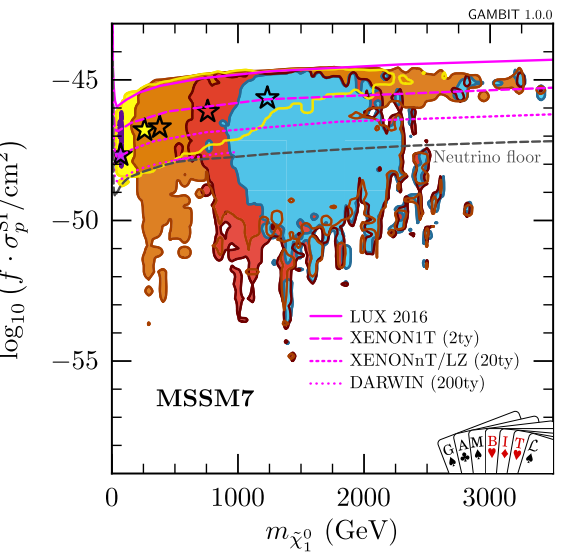

$\tilde{b}_{1}$ co-annihilation $h / Z$ funnel
Fig. 12 Spin-independent neutralino-proton scattering cross-sections in the MSSM7, rescaled by the fraction $f$ of the observed relic density predicted by each model. Left: profile likelihood, showing 68 and 95\% CL contours. Right: colour-coding shows mechanism(s) that allow models within the $95 \% \mathrm{CL}$ region of the profile likelihood to avoid exceeding the observed relic density of DM, corresponding to neutralino co-annihilation with charginos, stops or sbottoms, and resonant annihilation through the light or heavy Higgs funnels. Overplotted are

reveals that the lowest-mass points in the stop co-annihilation region remain unprobed, as do the chargino co-annihilation and $A / H$-funnel points. The $\tilde{t}_{1}-\tilde{\chi}_{1}^{0}$ mass difference is shown in the bottom panels of Fig. 9. Although this is of course small for the stop and sbottom co-annihilation region points, it is not, contrary to the chargino case, sharply peaked at sufficiently low values that decay products can be assumed to be hard to reconstruct at the LHC. This offers hope that LHC searches for compressed spectra (sensitive to smallish mass differences) can eventually tackle these models.
90\% CL constraints from LUX, [187], and projections for the reach of XENON1T after two years of exposure, XENONnT/LZ, assuming 1-3 years of data and an exposure of 20 tonne-years [239], and DARWIN, assuming 3-4 years of data and 200 tonne-years of exposure [240]. The dashed grey line indicates the "neutrino floor" where background events from coherent neutrino scattering start to limit the experimental sensitivity [241]. The exact placement of this limit is subject to several caveats; see [241] for further details

\subsection{Direct detection of dark matter}

In this section, we examine the preferred spin-independent (SI; Fig. 12) and spin-dependent (SD; Fig. 13) neutralinoproton scattering cross-sections in the MSSM7. Here we rescale the scattering cross-sections by the ratio $f$ of the predicted to the observed relic density, so as to ease comparison with various experimental limits and projections. Figure 12 shows that SI limits from direct detection are already highly constraining, with many models with high likelihoods lying 

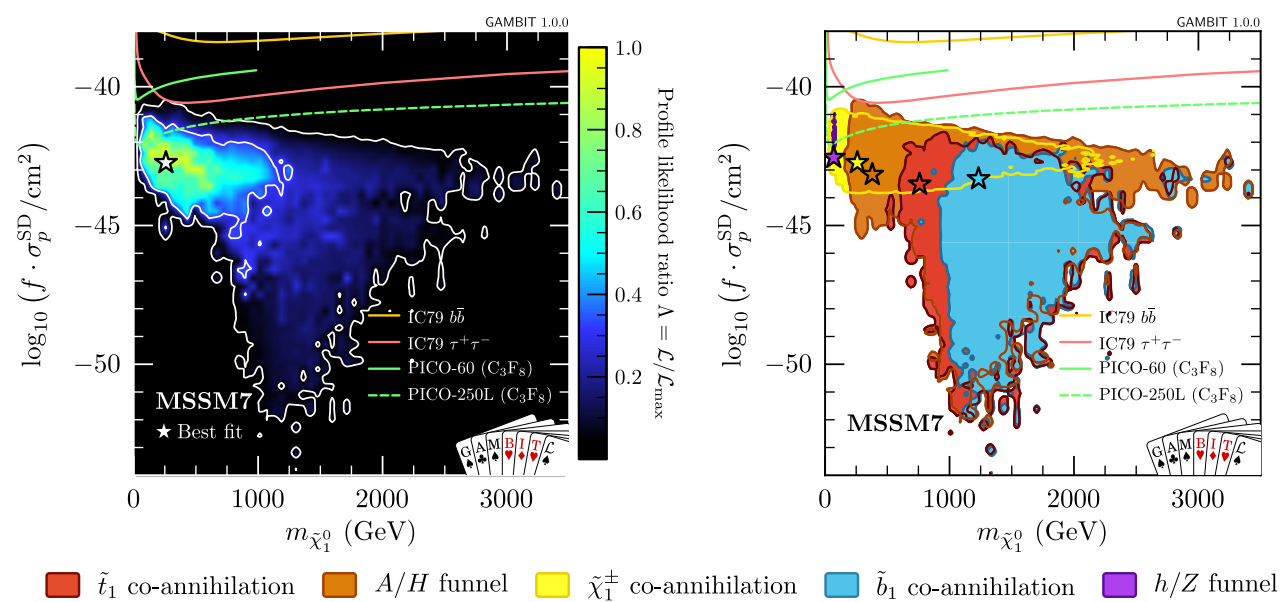

Fig. 13 Spin-dependent neutralino-proton scattering cross-sections in the MSSM7, rescaled by the fraction $f$ of the observed relic density predicted by each model. Left: profile likelihood, showing 68 and 95\% CL contours. Right: mechanism(s) that allow models within the $95 \%$ $\mathrm{CL}$ region of the profile likelihood to avoid exceeding the observed

just below the current sensitivity of LUX [187], and very soon to be probed by XENON1T [239] and its successors. Eventually, DARWIN [240] looks set to probe the entirety of the light Higgs funnel and the chargino co-annihilation region, as well as large parts of the heavy funnel and stop/sbottom co-annihilation regions.

In the SD sector, IceCube already constrains mixed gaugino-Higgsino models in the MSSM, as noted in Refs. [47, 175,247]. PICO [245] is not yet competitive for MSSM models, but its future upgrades appear set to make significant inroads into both Higgs funnels and the chargino co-annihilation region. However, it remains to be seen if XENON1T will probe such models on a shorter timescale. Future neutrino telescopes such as KM3NeT [248] and proposed upgrades to IceCube $[249,250]$ may also offer significantly improved sensitivity to models in the MSSM7, but to date the expected sensitivity to DM masses above $100 \mathrm{GeV}$ is not known. Whilst not particularly constraining in terms of SD proton scattering, LUX [251] already provides constraints on the SD neutralino-neutron cross-section, which are just beginning to touch on the allowed parameter space of the MSSM7 (not shown, but included in our scans via DDCalc [178]).

Although models exist down to SI and SD cross-sections of $10^{-55} \mathrm{~cm}^{2}$ in the stop/sbottom co-annihilation and $A / H$ funnel regions of our fits, the large cancellations required to produce such cross-sections may be spoilt by loop corrections $[254,255]$. This raises hope that future direct detection experiments will discover neutralino DM in the MSSM7 or a similar model. However, specific investigations in the MSSM7 suggest that this is not necessarily expected for all parameter combinations, so some parts of the parameter space should relic density of DM. Overplotted are 90\% CL constraints from IceCube $[47,184]$, assuming that dark matter annihilates exclusively via the $\bar{b} b$ or $\tau^{+} \tau^{-}$channel, PICO-60 [245], and projections for the reach of PICO-250 [246]

still be expected to lie well below any future sensitivity, even after applying higher-order corrections [172].

\subsection{Indirect detection of dark matter}

Let us finally address discovery prospects of the MSSM7 with indirect DM searches. To this end, we show in Fig. 14 the velocity-weighted annihilation cross section, $\sigma v$, in the limit of vanishing relative velocity of the annihilating DM particles, as a function of the lightest neutralino mass. We rescale this quantity by the square ${ }^{5}$ of the fraction $f$ of the calculated neutralino relic density to the observed DM abundance, thereby accounting for the possibility of the lightest neutralino making up only a fraction of DM. In the left panel, we show the profile likelihood, while in the right panel we indicate the mechanism(s) responsible for increasing the (co)annihilation rate in the early Universe, and hence decreasing the present neutralino relic density to or below the observed DM abundance. For comparison, we also indicate the same current and projected future limits from selected indirect detection experiments as in Fig. 21 of the companion paper [166], namely present Fermi-LAT [183] limits for $\bar{b} b$ and $\tau^{+} \tau^{-}$final states from observations of 15 dwarf galaxies, projected Fermi-LAT limits for $\bar{b} b$, and the projected sensitivity of the Chrerenkov Telescope Array (CTA) for $\bar{b} b$, assuming $500 \mathrm{~h}$ of Galactic halo observations [253].

Across almost the entire neutralino mass range, we find models within the $95 \%$ CL region of the profile likelihood that exhibit present-day annihilation rates above the canon-

\footnotetext{
5 Here we assume that all DM clumps just like neutralinos; see Sec. 4.4.3 of Ref. [166] for further discussion.
} 


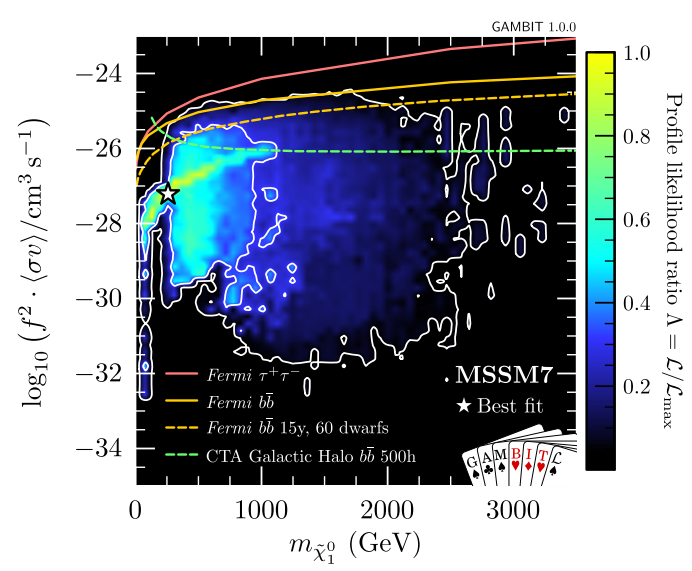

$\tilde{t}_{1}$ co-annihilation
$A / H$ funnel

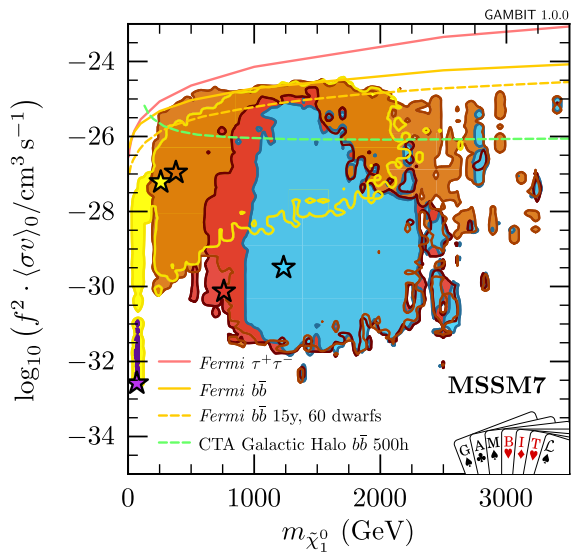

$\tilde{b}_{1}$ co-annihilation

$h / Z$ funnel
Fig. 14 Zero-velocity neutralino self-annihilation cross-sections in the MSSM7, rescaled by the square of the fraction $f$ of the observed relic density predicted by each model. Left: profile likelihood, showing 68 and $95 \%$ CL contours. Right: mechanism(s) that allow models within the $95 \% \mathrm{CL}$ region of the profile likelihood to avoid exceeding the observed relic density of DM. Overplotted are 95\% CL constraints from the search for dark matter annihilation in 15 dwarf spheroidal galaxies by the Fermi-LAT Collaboration [183]. These limits are based on 6 years

ical thermal value of $3 \times 10^{-26} \mathrm{~cm}^{3} \mathrm{~s}^{-1}$. Those models are a subset of the $A / H$ funnel region, where the pseudoscalar Higgs is almost exactly twice as heavy as the lightest neutralino, $m_{A} \simeq 2 \tilde{\chi}_{1}^{0}$. This leads to resonant enhancement of the annihilation rate as $v \rightarrow 0$, as is the case today - but not in the early Universe, where thermal effects mean that $v \neq 0$ in general. For some models in this part of the parameter space, current Fermi limits are already quite constraining. Projected Fermi limits, assuming 15 years of data on 60 dwarf galaxies (vs. 6 years and 15 dwarfs for the current limits), will start to cut into the (current) $68 \% \mathrm{CL}$ region. For neutralino masses above around $300 \mathrm{GeV}$, CTA will be even more sensitive than this. Large parts of the MSSM7 parameter space, however, will be impossible to probe with any planned indirect detection experiment; this includes, unfortunately, both the global best fit point of the MSSM7 and the best-fit points of all of the individual parameter regions corresponding to different mechanisms of lowering the relic density.

We emphasise that even though the CTA limits shown here are rather optimistic, in that they neglect the effect of systematic uncertainties [256], the above discussion somewhat underestimates the prospects of indirect DM searches. One reason is that we have neglected in our discussion other detection channels than gamma-rays, in particular charged cosmic rays. As discussed in some more detail in Sec. 4.4.3 of the companion paper [166], radiative corrections in particular, e.g. [257-259], as well as Sommerfeld enhancement [73,260-262], are further effects that we have not taken into account here. For parts of the parameter space this leads to increased annihilation rates and/or distinct spectral features, which are much easier to constrain or identify with experi- of Pass 8 data, and are given for two different assumed annihilation final states $\left(\bar{b} b\right.$ and $\left.\tau^{+} \tau^{-}\right)$. We also show the projected improvement in the $\bar{b} b$ channel after 15 years, if the number of known dwarfs were to quadruple in that time [252]. The final curve is the best-case projected sensitivity of the Cherenkov Telescope Array to annihilation in the Galactic halo, computed assuming $\bar{b} b$ final states, neglecting systematic errors, and assuming $500 \mathrm{~h}$ of observation [253]

ments than the featureless gamma-ray spectra from the final states that we have considered here. A full discussion of these effects, and their impact on indirect DM searches within the MSSM7, is beyond the scope of this study, although we plan to return to this in future work.

\section{Conclusions}

We have carried out an extensive global fit of the 7-parameter, weak-scale phenomenological MSSM, using the newlyreleased GAMBIT global fitting framework. Our fit takes into account updated experimental data, improved theoretical calculations and more advanced statistical sampling methods than previous studies of similar models. We have also considered leading uncertainties from the Standard Model, the dark matter halo of the Milky Way, and the quark content of the nucleon, fully scanning over the relevant parameters and profiling them out in the final fit. Finally, we have explored the full range of experimentally-allowed parameters, by allowing neutralinos to constitute any fraction of the observed cosmological dark matter.

The MSSM7 shows quite a rich selection of phenomenology across its parameter space, ranging from Higgsinodominated dark matter annihilating through co-annihilations with other Higgsinos in the early Universe, to resonant annihilation via the light and heavy Higgs funnels, to coannihilation of neutralinos with both light stops and sbottoms. We find a preference for light, Higgsino-dominated neutralinos, with $m_{\tilde{\chi}_{1}^{0}} \lesssim 750 \mathrm{GeV}$ at $68 \% \mathrm{CL}$ and $m_{\tilde{\chi}_{1}^{0}} \lesssim$ $2.5 \mathrm{TeV}$ at $95 \% \mathrm{CL}$. We have shown that stop/sbottom co- 
annihilation models lie just out of reach of current LHC searches, with stops and sbottoms as light at $500 \mathrm{GeV}$. This makes the prospects for probing at least some such models at the LHC in the near future quite promising. Both direct and indirect searches for dark matter place significant constraints on the allowed parameter ranges in the MSSM7, and the next generation of these experiments will probe large parts of the highest-likelihood areas of its parameter space.

The current study is essentially a starting point for detailed, modular scans of supersymmetric models defined at the weak scale with GAMBIT. GAMBIT's hierarchical model database already contains many generalisations of the MSSM7, which would themselves make very interesting targets for global analyses similar to this one.

To ensure reproducibility and encourage further exploration of our results, we provide a set of supplementary data online through Zenodo [170]. This includes all GAMBIT input files, generated likelihood samples and best-fit benchmarks for this paper.

Acknowledgements We thank Andrew Fowlie, Tomás Gonzalo, Julia Harz, Sebastian Hoof, Felix Kahlhoefer, James McKay, Roberto Ruiz, Roberto Trotta and Sebastian Wild for useful discussions, and Lucien Boland, Sean Crosby and Goncalo Borges of the Australian Centre of Excellence for Particle Physics at the Terascale for computing assistance and resources. We warmly thank the Casa Matemáticas Oaxaca, affiliated with the Banff International Research Station, for hospitality whilst part of this work was completed, and the staff at Cyfronet, for their always helpful supercomputing support. GAMBIT has been supported by STFC (UK; ST/K00414X/1, ST/P000762/1), the Royal Society (UK; UF110191), Glasgow University (UK; Leadership Fellowship), the Research Council of Norway (FRIPRO 230546/F20), NOTUR (Norway; NN9284K), the Knut and Alice Wallenberg Foundation (Sweden; Wallenberg Academy Fellowship), the Swedish Research Council (621-2014-5772), the Australian Research Council (CE110001004, FT130100018, FT140100244, FT160100274), The University of Sydney (Australia; IRCA-G162448), PLGrid Infrastructure (Poland), Red Española de Supercomputación (Spain; FI-2016-1-0021), Polish National Science Center (Sonata UMO-2015/17/D/ST2/03532), the Swiss National Science Foundation (PP00P2-144674), the European Commission Horizon 2020 Marie Skłodowska-Curie actions (H2020MSCA-RISE-2015-691164), the ERA-CAN+ Twinning Program (EU \& Canada), the Netherlands Organisation for Scientific Research (NWO-Vidi 680-47-532), the National Science Foundation (USA; DGE-1339067), the FRQNT (Québec) and NSERC/The Canadian TriAgencies Research Councils (BPDF-424460-2012).

Open Access This article is distributed under the terms of the Creative Commons Attribution 4.0 International License (http://creativecomm ons.org/licenses/by/4.0/), which permits unrestricted use, distribution, and reproduction in any medium, provided you give appropriate credit to the original author(s) and the source, provide a link to the Creative Commons license, and indicate if changes were made.

Funded by SCOAP ${ }^{3}$.

\section{References}

1. P. Fayet, The Supersymmetric Standard Model. Adv. Ser. Direct. High Energy Phys. 26, 397-454 (2016). arXiv:1506.08277
2. S. Dimopoulos, S. Raby, F. Wilczek, Supersymmetry and the scale of unification. Phys. Rev. D 24, 1681-1683 (1981)

3. E. Witten, Dynamical breaking of supersymmetry. Nucl. Phys. B 188, 513 (1981)

4. S. Dimopoulos, H. Georgi, Softly broken supersymmetry and SU(5). Nucl. Phys. B 193, 150-162 (1981)

5. N. Sakai, Naturalness in supersymmetric GUTs. Z. Phys. C 11, $153(1981)$

6. R.K. Kaul, P. Majumdar, Cancellation of quadratically divergent mass corrections in globally supersymmetric spontaneously broken gauge theories. Nucl. Phys. B 199, 36 (1982)

7. S. Weinberg, Implications of dynamical symmetry breaking. Phys. Rev. D 13, 974-996 (1976)

8. S. Weinberg, Implications of dynamical symmetry breaking: an addendum. Phys. Rev. D 19, 1277-1280 (1979)

9. E. Gildener, Gauge symmetry hierarchies. Phys. Rev. D 14, 1667 (1976)

10. L. Susskind, Dynamics of spontaneous symmetry breaking in the Weinberg-Salam theory. Phys. Rev. D 20, 2619-2625 (1979)

11. G. 't Hooft, C. Itzykson, et. al., Recent Developments in Gauge Theories. in Proceedings, Nato Advanced Study Institute, Cargese, France, August 26-September 8, 1979, vol 59 (NATO Sci. Ser. B, 1980), pp. 1-438

12. J.R. Ellis, S. Kelley, D.V. Nanopoulos, Probing the desert using gauge coupling unification. Phys. Lett. B 260, 131-137 (1991)

13. P. Langacker, M.-X. Luo, Implications of precision electroweak experiments for $M_{t}, \rho_{0}, \sin ^{2} \theta_{W}$ and grand unification. Phys. Rev. D 44, 817-822 (1991)

14. U. Amaldi, W. de Boer, H. Furstenau, Comparison of grand unified theories with electroweak and strong coupling constants measured at LEP. Phys. Lett. B 260, 447-455 (1991)

15. F. Anselmo, L. Cifarelli, A. Peterman, A. Zichichi, The effective experimental constraints on M(SUSY) and M(GUT). Nuovo Cim. A 104, 1817-1834 (1991)

16. J.R. Ellis, J.S. Hagelin, D.V. Nanopoulos, K.A. Olive, M. Srednicki, Supersymmetric relics from the Big Bang. Nucl. Phys. B 238, 453-476 (1984)

17. G. Jungman, M. Kamionkowski, K. Griest, Supersymmetric dark matter. Phys. Rep. 267, 195-373 (1996). arXiv:hep-ph/9506380

18. ATLAS Collaboration, G. Aad et. al., Observation of a new particle in the search for the standard model Higgs boson with the ATLAS detector at the LHC. Phys. Lett. B 716, 1-29 (2012). arXiv: 1207.7214

19. S. Chatrchyan, V. Khachatryan et al., Observation of a new boson at a mass of $125 \mathrm{GeV}$ with the CMS experiment at the LHC. Phys. Lett. B 716, 30-61 (2012). arXiv: 1207.7235

20. E. Bagnaschi, G.F. Giudice, P. Slavich, A. Strumia, Higgs mass and unnatural supersymmetry. JHEP 09, 092 (2014). arXiv: 1407.4081

21. J. Pardo Vega, G. Villadoro, SusyHD: Higgs mass determination in supersymmetry. JHEP 07, 159 (2015). arXiv: 1504.05200

22. G. Lee, C.E.M. Wagner, Higgs bosons in heavy supersymmetry with an intermediate $\mathrm{m}_{A}$. Phys. Rev. D 92, 075032 (2015). arXiv: 1508.00576

23. P. Draper, H. Rzehak, A review of Higgs mass calculations in supersymmetric models. Phys. Rep. 619, 1-24 (2016). arXiv: 1601.01890

24. H. Bahl, W. Hollik, Precise prediction for the light MSSM Higgs boson mass combining effective field theory and fixed-order calculations. Eur. Phys. J. C 76, 499 (2016). arXiv:1608.01880

25. P. Athron, J.-H. Park, T. Steudtner, D. Stöckinger, A. Voigt, Precise Higgs mass calculations in (non-)minimal supersymmetry at both high and low scales. JHEP 079, 01 (2017). arXiv:1609.00371

26. E. Bagnaschi, J. Pardo Vega, P. Slavich, Improved determination of the Higgs mass in the MSSM with heavy superpartners. Eur. Phys. J. C77, 334 (2017). arXiv: 1703.08166 
27. F. Staub, W. Porod, Improved predictions for intermediate and heavy Supersymmetry in the MSSM and beyond. arXiv: 1703.03267

28. S. Paßehr, G. Weiglein, Two-loop top and bottom Yukawa corrections to the Higgs-boson masses in the complex MSSM. arXiv: 1705.07909

29. H. Bahl, S. Heinemeyer, W. Hollik, G. Weiglein, Reconciling EFT and hybrid calculations of the light MSSM Higgs-boson mass. arXiv: 1706.00346

30. R.K. Barman, B. Bhattacherjee, et. al., Status of MSSM Higgs sector after ICHEP 2016. arXiv:1608.02573

31. P. Bechtle, H.E. Haber et al., The light and heavy Higgs interpretation of the MSSM. Eur. Phys. J. C 77, 67 (2017). arXiv: 1608.00638

32. M. Carena, J. Ellis, J.S. Lee, A. Pilaftsis, C.E.M. Wagner, CP violation in heavy MSSM Higgs scenarios. JHEP 02, 123 (2016). arXiv: 1512.00437

33. B. Bhattacherjee, M. Chakraborti, A. Chakraborty, U. Chattopadhyay, D.K. Ghosh, Status of the $98-125 \mathrm{GeV}$ Higgs bosons scenario with updated LHC-8 data. Phys. Rev. D 93, 075004 (2016). arXiv:1511.08461

34. J. Baglio, A. Djouadi, J. Quevillon, Prospects for Higgs physics at energies up to $100 \mathrm{TeV}$. Rep. Prog. Phys. 79, 116201 (2016). arXiv: 1511.07853

35. S. Heinemeyer, C. Schappacher, Neutral Higgs boson production at $e^{+} e^{-}$colliders in the complex MSSM: a full one-loop analysis. Eur. Phys. J. C 76, 220 (2016). arXiv: 1511.06002

36. E. Arganda, J. Guasch, W. Hollik, S. Penaranda, Discriminating between SUSY and non-SUSY Higgs sectors through the ratio $H \rightarrow b \bar{b} / H \rightarrow \tau^{+} \tau^{-}$with a $125 \mathrm{GeV}$ Higgs boson. Eur. Phys. J. C 76, 286 (2016). arXiv: 1506.08462

37. L. Wu, J.M. Yang, C.-P. Yuan, M. Zhang, Higgs self-coupling in the MSSM and NMSSM after the LHC Run 1. Phys. Lett. B 747, 378-389 (2015). arXiv:1504.06932

38. H. Mantler, M. Wiesemann, Hadronic Higgs production through $\mathrm{NLO}+\mathrm{PS}$ in the SM, the 2HDM and the MSSM. Eur. Phys. J. C 75, 257 (2015). arXiv: 1504.06625

39. B. Bhattacherjee, A. Chakraborty, A. Choudhury, Status of the MSSM Higgs sector using global analysis and direct search bounds, and future prospects at the high luminosity LHC. Phys. Rev. D 92, 093007 (2015). arXiv: 1504.04308

40. A. Djouadi, L. Maiani, A. Polosa, J. Quevillon, V. Riquer, Fully covering the MSSM Higgs sector at the LHC. JHEP 06, 168 (2015). arXiv: 1502.05653

41. S. Profumo, T. Stefaniak, Alignment without decoupling: the portal to light dark matter in the MSSM. Phys. Rev. D 94, 095020 (2016). arXiv: 1608.06945

42. D.P. Roy, SUSY dark matter in universal and nonuniversal gaugino mass models. arXiv: 1608.03062

43. J. Harz, B. Herrmann, M. Klasen, K. Kovarik, P. Steppeler, Theoretical uncertainty of the supersymmetric dark matter relic density from scheme and scale variations. Phys. Rev. D 93, 114023 (2016). arXiv: 1602.08103

44. Q. Riffard, F. Mayet, G. Bélanger, M.H. Genest, D. Santos, Extracting constraints from direct detection searches of supersymmetric dark matter in the light of null results from the LHC in the squark sector. Phys. Rev. D 93, 035022 (2016). arXiv:1602.01030

45. M. van Beekveld, W. Beenakker, S. Caron, R. Ruiz de Austri, The case for $100 \mathrm{GeV}$ bino dark matter: a dedicated LHC tri-lepton search. JHEP 04, 154 (2016). arXiv: 1602.00590

46. M. Beneke, A. Bharucha et al., Relic density of wino-like dark matter in the MSSM. JHEP 03, 119 (2016). arXiv:1601.04718

47. IceCube Collaboration, M.G. Aartsen et. al., Improved limits on dark matter annihilation in the Sun with the 79-string IceCube detector and implications for supersymmetry. JCAP04, 022 (2016). arXiv: 1601.00653
48. A. Berlin, D.S. Robertson, M.P. Solon, Bino variations: effective field theory methods for dark matter direct detection. Phys. Rev. D 93, 095008 (2016). arXiv: 1511.05964

49. J. Cao, Y. He, L. Shang, W. Su, Y. Zhang, Testing the light dark matter scenario of the MSSM at the LHC. JHEP 03, 207 (2016). arXiv: 1511.05386

50. J. Harz, B. Herrmann, M. Klasen, K. Kovarik, P. Steppeler, precise prediction of the dark matter relic density within the MSSM. PoSEPS-HEP2015, 410 (2015). arXiv: 1510.06295

51. K. Hamaguchi, K. Ishikawa, Prospects for Higgs- and Zresonant neutralino dark matter. Phys. Rev. D 93, 055009 (2016). arXiv: 1510.05378

52. A.L. Erickcek, K. Sinha, S. Watson, Bringing isolated dark matter out of isolation: late-time reheating and indirect detection. Phys. Rev. D 94, 063502 (2016). arXiv: 1510.04291

53. J. Ellis, J.L. Evans, F. Luo, K.A. Olive, Scenarios for Gluino Coannihilation. JHEP 02, 071 (2016). arXiv: 1510.03498

54. J. Bramante, N. Desai et al., Towards the final word on neutralino dark matter. Phys. Rev. D 93, 063525 (2016). arXiv: 1510.03460

55. M. Baumgart, V. Vaidya, Semi-inclusive wino and higgsino annihilation to LL. JHEP 03, 213 (2016). arXiv: 1510.02470

56. T. Bringmann, A.J. Galea, P. Walia, Leading QCD corrections for indirect dark matter searches: a fresh look. Phys. Rev. D 93, 043529 (2016)

57. H. Eberl, V.C. Spanos, GravitinoPack and decays of supersymmetric metastable particles. Comput. Phys. Commun. 202, 310-325 (2016). arXiv: 1509.09159

58. K. Freese, A. Lopez, N.R. Shah, B. Shakya, MSSM A-funnel and the Galactic center excess: prospects for the LHC and direct detection experiments. JHEP 04, 059 (2016). arXiv: 1509.05076

59. M. Berggren, A. Cakir et al., Non-simplified SUSY: $\tilde{\tau}$ coannihilation at LHC and ILC. Eur. Phys. J. C 76, 183 (2016). arXiv: 1508.04383

60. S. Raby, Long-lived gluinos and stable axinos. Phys. Rev. Lett. 115, 231801 (2015). arXiv:1508.04373

61. L. Roszkowski, S. Trojanowski, K. Turzynski, Axino dark matter with low reheating temperature. JHEP 11, 139 (2015). arXiv:1507.06164

62. J. Bramante, P.J. Fox et al., Relic neutralino surface at a $100 \mathrm{TeV}$ collider. Phys. Rev. D 91, 054015 (2015). arXiv:1412.4789

63. G. Arcadi, L. Covi, M. Nardecchia, Gravitino dark matter and low-scale baryogenesis. Phys. Rev. D 92, 115006 (2015). arXiv: 1507.05584

64. A. Achterberg, M. van Beekveld, W. Beenakker, S. Caron, Comparing Galactic center MSSM dark matter solutions to the reticulum II gamma-ray data. JCAP 1512, 013 (2015). arXiv: 1507.04644

65. A. Arbey, M. Battaglia, L. Covi, J. Hasenkamp, F. Mahmoudi, LHC constraints on gravitino dark matter. Phys. Rev. D 92, 115008 (2015). arXiv: 1505.04595

66. A. Crivellin, M. Hoferichter, M. Procura, L.C. Tunstall, Dark matter: connecting LHC searches to direct detection. in Gravitation: 100 years after GR. Proceedings of the 50th Rencontres de Moriond, La Thuile, Italy, March 21-28, 2015. (2015), pp. 237-242. arXiv: 1505.02314

67. M. Ibe, S. Matsumoto, S. Shirai, T.T. Yanagida, Wino dark matter in light of the AMS-02 2015 data. Phys. Rev. D 91, 111701 (2015). arXiv: 1504.05554

68. A. Arbey, M. Battaglia, F. Mahmoudi, The Higgs boson, supersymmetry and dark matter: relations and perspectives. Ann. Phys. 528, 179-186 (2016). arXiv:1504.05091

69. J. Hisano, K. Ishiwata, N. Nagata, QCD effects on direct detection of wino dark matter. JHEP 06, 097 (2015). arXiv:1504.00915

70. N. Nagata, H. Otono, S. Shirai, Probing bino-gluino coannihilation at the LHC. Phys. Lett. B 748, 24-29 (2015). arXiv: 1504.00504 
71. J. Ellis, F. Luo, K.A. Olive, Gluino coannihilation revisited. JHEP 09, 127 (2015). arXiv: 1503.07142

72. A. Crivellin, M. Hoferichter, M. Procura, L.C. Tunstall, Light stops, blind spots, and isospin violation in the MSSM. JHEP 07, 129 (2015). arXiv: 1503.03478

73. M.E. Cabrera-Catalan, S. Ando, C. Weniger, F. Zandanel, Indirect and direct detection prospect for $\mathrm{TeV}$ dark matter in the nine parameter MSSM. Phys. Rev. D 92, 035018 (2015). arXiv: 1503.00599

74. A. Berlin, S. Gori, T. Lin, L.-T. Wang, Pseudoscalar portal dark matter. Phys. Rev. D 92, 015005 (2015). arXiv: 1502.06000

75. A. Achterberg, S. Amoroso et al., A description of the Galactic center excess in the minimal supersymmetric standard model. JCAP 1508, 006 (2015). arXiv:1502.05703

76. L. Aparicio, M. Cicoli et al., Non-thermal CMSSM with a 125 GeV Higgs. JHEP 05, 098 (2015). arXiv:1502.05672

77. G.L. Kane, P. Kumar, B.D. Nelson, B. Zheng, Dark matter production mechanisms with a nonthermal cosmological history: a classification. Phys. Rev. D 93, 063527 (2016). arXiv:1502.05406

78. M. Baumgart, I.Z. Rothstein, V. Vaidya, Constraints on galactic wino densities from gamma ray lines. JHEP 04, 106 (2015). arXiv: 1412.8698

79. S. Liebler, S. Profumo, T. Stefaniak, Light stop mass limits from Higgs rate measurements in the MSSM: is MSSM electroweak baryogenesis still alive after all? JHEP 04, 143 (2016). arXiv: 1512.09172

80. M. Dhuria, C. Hati, U. Sarkar, Moduli induced cogenesis of baryon asymmetry and dark matter. Phys. Lett. B 756, 376-383 (2016). arXiv: 1508.04144

81. G. Barenboim, W.-I. Park, Peccei-Quinn field for inflation, baryogenesis, dark matter, and much more. Phys. Lett. B 756, 317-322 (2016). arXiv: 1508.00011

82. W.G. Hollik, A new view on vacuum stability in the MSSM. JHEP 08, 126 (2016). arXiv: 1606.08356

83. J. Ellis, M.A.G. Garcia, D.V. Nanopoulos, K.A. Olive, Phenomenological aspects of no-scale inflation models. JCAP 1510, 003 (2015). arXiv: 1503.08867

84. F.S. Queiroz, K. Sinha, W. Wester, Rich tapestry: supersymmetric axions, dark radiation, and inflationary reheating. Phys. Rev. D 90, 115009 (2014). arXiv: 1407.4110

85. S. Kasuya, F. Takahashi, Flat direction inflation with running kinetic term and baryogenesis. Phys. Lett. B 736, 526-532 (2014). arXiv: 1405.4125

86. C. Pallis, Linking starobinsky-type inflation in no-scale supergravity to MSSM. JCAP 1404, 024 (2014). arXiv:1312.3623

87. M. Bose, M. Dine, P. Draper, Moduli or not. Phys. Rev. D 88, 023533 (2013). arXiv: 1305.1066

88. C. Pallis, Q. Shafi, Update on minimal supersymmetric hybrid inflation in light of PLANCK. Phys. Lett. B 725, 327-333 (2013). arXiv: 1304.5202

89. L. Wang, E. Pukartas, A. Mazumdar, Visible sector inflation and the right thermal history in light of Planck data. JCAP 1307, 019 (2013). arXiv:1303.5351

90. C. Boehm, J. Da Silva, A. Mazumdar, E. Pukartas, Probing the supersymmetric inflaton and dark matter link via the CMB, LHC and XENON1T experiments. Phys. Rev. D 87, 023529 (2013). arXiv: 1205.2815

91. A. Kobakhidze, M. Talia, L. Wu, Probing the MSSM explanation of the muon g-2 anomaly in dark matter experiments and at a $100 \mathrm{TeV} p p$ collider. Phys. Rev. D 95, 055023 (2017). arXiv: 1608.03641

92. P. Athron, M. Bach et al., GM2Calc: precise MSSM prediction for (g-2) of the muon. Eur. Phys. J. C 76, 62 (2016). arXiv:1510.08071

93. J. Hisano, D. Kobayashi, W. Kuramoto, T. Kuwahara, Nucleon electric dipole moments in high-scale supersymmetric models. JHEP 11, 085 (2015). arXiv:1507.05836
94. K. Harigaya, T.T. Yanagida, N. Yokozaki, Muon $g-2$ in focus point SUSY. Phys. Rev. D 92, 035011 (2015). arXiv:1505.01987

95. M. Bach, J.-H. Park, D. Stöckinger, H. Stöckinger-Kim, Large muon $(g-2)$ with TeV-scale SUSY masses for $\tan \beta \rightarrow \infty$. JHEP 10, 026 (2015). arXiv:1504.05500

96. J. Chakrabortty, A. Choudhury, S. Mondal, Non-universal Gaugino mass models under the lamppost of muon (g-2). JHEP 07, 038 (2015). arXiv: 1503.08703

97. K. Kowalska, L. Roszkowski, E.M. Sessolo, A.J. Williams, GUTinspired SUSY and the muon $g-2$ anomaly: prospects for LHC 14 TeV. JHEP 06, 020 (2015). arXiv:1503.08219

98. A. Aboubrahim, T. Ibrahim, P. Nath, Neutron electric dipole moment and probe of PeV scale physics. Phys. Rev. D 91, 095017 (2015). arXiv:1503.06850

99. K. De Causmaecker, B. Fuks et al., General squark flavour mixing: constraints, phenomenology and benchmarks. JHEP 11, 125 (2015). arXiv: 1509.05414

100. J.A. Evans, D. Shih, A. Thalapillil, Chiral flavor violation from extended gauge mediation. JHEP 07, 040 (2015). arXiv: 1504.00930

101. K. Kowalska, Phenomenological MSSM in light of new $13 \mathrm{TeV}$ LHC data. Eur. Phys. J. C 76, 684 (2016). arXiv:1608.02489

102. M. Cannoni, J. Ellis, M.E. Gómez, S. Lola, R. Ruiz de Austri, Supersymmetry searches in GUT models with non-universal scalar masses. JCAP 1603, 041 (2016). arXiv: 1511.06205

103. J. Berger, M.W. Cahill-Rowley et al., $C P$-violating phenomenological MSSM. Phys. Rev. D 93, 035017 (2016). arXiv: 1510.08840

104. G. Bertone, F. Calore et al., Global analysis of the pMSSM in light of the Fermi $\mathrm{GeV}$ excess: prospects for the LHC Run-II and astroparticle experiments. JCAP 1604, 037 (2016). arXiv: 1507.07008

105. E.A. Bagnaschi, O. Buchmueller et al., Supersymmetric dark matter after LHC run 1. Eur. Phys. J. C 75, 500 (2015). arXiv: 1508.01173

106. S.S. AbdusSalam, L. Velasco-Sevilla, Where to look for natural supersymmetry. Phys. Rev. D 94, 035026 (2016). arXiv:1506.02499

107. K.J. de Vries et al., The pMSSM10 after LHC Run 1. Eur. Phys. J. C 75, 422 (2015). arXiv: 1504.03260

108. C. Han, K.-i. Hikasa, L. Wu, J. M. Yang, and Y. Zhang, Status of CMSSM in light of current LHC Run-2 and LUX data, arXiv: 1612.02296

109. J. Ellis, J.L. Evans, A. Mustafayev, N. Nagata, K.A. Olive, The super-GUT CMSSM revisited. Eur. Phys. J. C 76, 592 (2016). arXiv: 1608.05370

110. R. Kitano, R. Motono, M. Nagai, MSSM without free parameters. Phys. Rev. D 94, 115016 (2016). arXiv:1605.08227

111. S. Banerjee, G. Bélanger, B. Mukhopadhyaya, P.D. Serpico, Signatures of sneutrino dark matter in an extension of the CMSSM. JHEP 07, 095 (2016). arXiv: 1603.08834

112. J. Ellis, J.L. Evans et al., Beyond the CMSSM without an accelerator: proton decay and direct dark matter detection. Eur. Phys. J. C 76, 8 (2016). arXiv: 1509.08838

113. W. Abdallah, S. Khalil, MSSM Dark Matter in Light of Higgs and LUX Results. Adv. High Energy Phys. 2016, 5687463 (2016). arXiv: 1509.07031

114. B. Kaufman, P. Nath, B.D. Nelson, A.B. Spisak, Light stops and observation of supersymmetry at LHC RUN-II. Phys. Rev. D 92, 095021 (2015). arXiv:1509.02530

115. P. Bechtle, J.E. Camargo-Molina et al., Killing the cMSSM softly. Eur. Phys. J. C 76, 96 (2016). arXiv: 1508.05951

116. K. Kowalska, L. Roszkowski, E.M. Sessolo, S. Trojanowski, A.J. Williams, Looking for supersymmetry: 1 Tev WIMP and the power of complementarity in LHC and dark matter searches, in Proceedings, 50th Rencontres de Moriond, QCD and high energy 
interactions: La Thuile, Italy, March 21-28, 2015 (2015), pp. 195 198. arXiv: 1507.07446

117. O. Buchmueller, M. Citron et al., Collider interplay for supersymmetry, higgs and dark matter. Eur. Phys. J. C 75, 469 (2015). arXiv:1505.04702. [Erratum: Eur. Phys. J. C 76(4), 190 (2016)]

118. N. Karagiannakis, G. Lazarides, C. Pallis, Probing the hyperbolic branch/focus point region of the constrained minimal supersymmetric standard model with generalized Yukawa quasiunification. Phys. Rev. D 92, 085018 (2015). arXiv: 1503.06186

119. B. Dutta, Y. Mimura, Is electroweak symmetry breaking still natural in the MSSM? arXiv:1608.07195

120. M.E. Cabrera, J.A. Casas, A. Delgado, S. Robles, R. Ruiz de Austri, Naturalness of MSSM dark matter. JHEP 08, 058 (2016). arXiv: 1604.02102

121. G.G. Ross, K. Schmidt-Hoberg, F. Staub, On the MSSM Higgsino mass and fine tuning. Phys. Lett. B 759, 110-114 (2016). arXiv: 1603.09347

122. F. Wang, J.M. Yang, Y. Zhang, Radiative natural SUSY spectrum from deflected AMSB scenario with messenger-matter interactions. JHEP 04, 177 (2016). arXiv:1602.01699

123. J. Fan, R. Krall, D. Pinner, M. Reece, J.T. Ruderman, Stealth Supersymmetry Simplified. JHEP 07, 016 (2016). arXiv:1512.05781

124. H. Baer, V. Barger, M. Savoy, Upper bounds on sparticle masses from naturalness or how to disprove weak scale supersymmetry. Phys. Rev. D 93, 035016 (2016). arXiv:1509.02929

125. G. Bhattacharyya, T.T. Yanagida, N. Yokozaki, Focus point gauge mediation with incomplete adjoint messengers and gauge coupling unification. Phys. Lett. B 749, 82-87 (2015). arXiv: 1506.05962

126. M. Fabbrichesi, A. Urbano, Naturalness redux: the case of the neutrino seesaw mechanism. Phys. Rev. D 92, 015028 (2015). arXiv: 1504.05403

127. K.J. Bae, H. Baer, V. Barger, M.R. Savoy, H. Serce, Supersymmetry with radiatively-driven naturalness: implications for WIMP and axion searches. Symmetry 7, 788-814 (2015). arXiv: 1503.04137

128. B.A. Ovrut, A. Purves, S. Spinner, The minimal SUSY $B-L$ model: from the unification scale to the LHC. JHEP 06, 182 (2015). arXiv: 1503.01473

129. G. Du, T. Li, D.V. Nanopoulos, S. Raza, Super-natural MSSM. Phys. Rev. D 92, 025038 (2015). arXiv: 1502.06893

130. S. Zheng, Focus point in dark matter selected high-scale supersymmetry. JHEP 03, 098 (2015). arXiv:1501.05698. [Erratum: JHEP 04, 104 (2015)]

131. S.A.R. Ellis, J.D. Wells, High-scale supersymmetry, the Higgs mass and gauge unification. arXiv: 1706.00013

132. M. Crispim Romão, A. Karozas, S.F. King, G.K. Leontaris, A.K. Meadowcroft, MSSM from F-theory SU(5) with Klein monodromy. Phys. Rev. D 93, 126007 (2016). arXiv:1512.09148

133. D. Bourilkov, Strong coupling running. gauge coupling unification and the scale of new physics. JHEP 11, 117 (2015). arXiv: 1508.04176

134. C.S. Aulakh, New minimal SO(10) GUT: a theory for all epochs. Pramana 86, 207-221 (2016). arXiv: 1506.05850

135. Z. Berezhiani, M. Chianese, G. Miele, S. Morisi, Chances for SUSY-GUT in the LHC epoch. JHEP 08, 083 (2015). arXiv: 1505.04950

136. A. Barr, J. Liu, Analysing parameter space correlations of recent $13 \mathrm{TeV}$ gluino and squark searches in the pMSSM. Eur. Phys. J. C 77, 202 (2017). arXiv: 1608.05379

137. W. Beenakker, C. Borschensky, M. Krämer, A. Kulesza, E. Laenen, NNLL-fast: predictions for coloured supersymmetric particle production at the LHC with threshold and Coulomb resummation. JHEP 12, 133 (2016). arXiv:1607.07741
138. A. Barr, J. Liu, First interpretation of $13 \mathrm{TeV}$ supersymmetry searches in the pMSSM. arXiv:1605.09502

139. S. Caron, J.S. Kim, K. Rolbiecki, R. Ruiz de Austri, B. Stienen, The BSM-AI project: SUSY-AIG generalizing LHC limits on supersymmetry with machine learning. Eur. Phys. J. C 77, 257 (2017). arXiv: 1605.02797

140. H. Baer, V. Barger, M. Savoy, X. Tata, Multichannel assault on natural supersymmetry at the high luminosity LHC. Phys. Rev. D 94, 035025 (2016). arXiv: 1604.07438

141. J. Dutta, P. Konar, S. Mondal, B. Mukhopadhyaya, S.K. Rai, A revisit to a compressed supersymmetric spectrum with $125 \mathrm{GeV}$ Higgs. JHEP 01, 051 (2016). arXiv:1511.09284

142. J. Kalinowski, SUSY with R-symmetry: confronting EW precision observables and LHC constraints. Acta Phys. Polon 47, 203 (2016). arXiv: 1510.06652

143. J. Heisig, A. Lessa, L. Quertenmont, Simplified models for exotic BSM searches. JHEP 12, 087 (2015). arXiv:1509.00473

144. P. Stengel, X. Tata, Same-sign Higgsino production at the CERN LHC: how not to hunt for natural supersymmetry. Phys. Rev. D 92, 115024 (2015). arXiv:1507.06726

145. P. Bechtle, S. Heinemeyer, O. Stål, T. Stefaniak, G. Weiglein, Applying exclusion likelihoods from LHC searches to extended Higgs sectors. Eur. Phys. J. C 75, 421 (2015). arXiv:1507.06706

146. T. Han, S. Su, Y. Wu, B. Zhang, H. Zhang, Sbottom discovery via mixed decays at the LHC. Phys. Rev. D 92, 115009 (2015). arXiv: 1507.04006

147. P. Bechtle, T. Plehn, C. Sander, Supersymmetry, in The Large Hadron Collider: Harvest of Run 1 ed. by T. Schörner-Sadenius (2015), pp. 421-462. arXiv:1506.03091

148. A. Arbey, M. Battaglia, F. Mahmoudi, Monojet searches for MSSM simplified models. Phys. Rev. D 94, 055015 (2016). arXiv: 1506.02148

149. G. Belanger, D. Ghosh, R. Godbole, S. Kulkarni, Light stop in the MSSM after LHC Run 1. JHEP 09, 214 (2015). arXiv:1506.00665

150. H. Abe, J. Kawamura, Y. Omura, LHC phenomenology of natural MSSM with non-universal gaugino masses at the unification scale. JHEP 08, 089 (2015). arXiv: 1505.03729

151. J.L. Feng, S. Iwamoto, Y. Shadmi, S. Tarem, Long-lived sleptons at the LHC and a $100 \mathrm{TeV}$ proton collider. JHEP 12, 166 (2015). arXiv:1505.02996

152. J.S. Gainer, K.T. Matchev, M. Park, The hierarchy solution to the LHC inverse problem. JHEP 06, 014 (2015). arXiv:1504.03689

153. A. Arbey et al., Physics at the $e^{+} e^{-}$linear collider. Eur. Phys. J. C 75, 371 (2015). arXiv:1504.01726

154. M. Backović, A. Mariotti, M. Spannowsky, Signs of tops from highly mixed stops. JHEP 06, 122 (2015). arXiv:1504.00927

155. S. Jung, H.-S. Lee, Untracked signals of supersymmetry at the LHC. arXiv:1503.00414

156. P. Grothaus, S.P. Liew, K. Sakurai, A closer look at a hint of SUSY at the 8 TeV LHC. JHEP 05, 133 (2015). arXiv: 1502.05712

157. A. Berlin, T. Lin, M. Low, L.-T. Wang, Neutralinos in vector boson fusion at high energy colliders. Phys. Rev. D 91, 115002 (2015). arXiv: 1502.05044

158. C. Han, D. Kim, S. Munir, M. Park, Accessing the core of naturalness, nearly degenerate higgsinos, at the LHC. JHEP 04, 132 (2015). arXiv:1502.03734

159. S. Fichet, B. Herrmann, Y. Stoll, Tasting the SU(5) nature of supersymmetry at the LHC. JHEP 05, 091 (2015). arXiv:1501.05307

160. H.P. Nilles, Supersymmetry, supergravity and particle physics. Phys. Rep. 110, 1-162 (1984)

161. D. Matalliotakis, H.P. Nilles, Implications of nonuniversality of soft terms in supersymmetric grand unified theories. Nucl. Phys. B 435, 115-128 (1995). arXiv:hep-ph/9407251

162. M. Olechowski, S. Pokorski, Electroweak symmetry breaking with nonuniversal scalar soft terms and large tan beta solutions. Phys. Lett. B 344, 201-210 (1995). arXiv:hep-ph/9407404 
163. V. Berezinsky, A. Bottino et al., Neutralino dark matter in supersymmetric models with nonuniversal scalar mass terms. Astropart. Phys. 5, 1-26 (1996). arXiv:hep-ph/9508249

164. M. Drees, M.M. Nojiri, D.P. Roy, Y. Yamada, Light Higgsino dark matter, Phys. Rev. D 56, 276-290 (1997). arXiv: hep-ph/9701219. [Erratum: Phys. Rev. D 64, 039901 (2001)]

165. P. Nath, R.L. Arnowitt, Nonuniversal soft SUSY breaking and dark matter. Phys. Rev. D 56, 2820-2832 (1997). arXiv:hep-ph/9701301

166. GAMBIT Collaboration, P. Athron, C. Balázs, et. al., Global fits of GUT-scale SUSY models with GAMBIT. arXiv:1705.07935

167. MSSM Working Group, Djouadi, A. et. al., The Minimal supersymmetric standard model: group summary report. in GDR (Groupement De Recherche)-Supersymetrie, Montpellier, France, April 15-17, 1998 (1998). arXiv: hep-ph/9901246

168. GAMBIT Collaboration, P. Athron, C. Balazs, et. al., GAMBIT: the global and modular beyond-the-standard-model inference tool. arXiv: 1705.07908

169. GAMBIT Scanner Workgroup, G.D. Martinez, J.McKay, et. al., Comparison of statistical sampling methods with ScannerBit, the GAMBIT scanning module. arXiv:1705.07959

170. GAMBIT Collaboration, Supplementary data: a global fit of the MSSM with GAMBIT (2017). arXiv:1705.07917. :DOIurl10.5281/zenodo.801640

171. L. Bergström, P. Gondolo, Limits on direct detection of neutralino dark matter from $b \rightarrow s \gamma$ decays. Astropart. Phys. 5, 263-278 (1996). arXiv:hep-ph/9510252

172. V. Mandic, A. Pierce, P. Gondolo, H. Murayama, The Lower bound on the neutralino nucleon cross-section. arXiv:hep-ph/0008022

173. P. Gondolo, J. Edsjö et al., DarkSUSY: computing supersymmetric dark matter properties numerically. JCAP 7, 8 (2004). arXiv:astro-ph/0406204

174. M. Berg, J. Edsjö, P. Gondolo, E. Lundström, S. Sjörs, Neutralino dark matter in BMSSM effective theory. JCAP 8, 35 (2009). arXiv:0906.0583

175. P. Scott, C. Savage, J. Edsjö, the IceCube Collaboration, R. Abbasi et al., Use of event-level neutrino telescope data in global fits for theories of new physics. JCAP 11, 57 (2012). arXiv:1207.0810

176. ANTARES Collaboration, S. Adrian-Martinez et. al., First results on dark matter annihilation in the Sun using the ANTARES neutrino telescope. JCAP 1311, 032 (2013). arXiv:1302.6516

177. B.C. Allanach et al., SUSY Les Houches accord 2. Comput. Phys. Commun. 180, 8-25 (2009). arXiv:0801.0045

178. GAMBIT Dark Matter Workgroup, T. Bringmann, J. Conrad, et. al., DarkBit: a GAMBIT module for computing dark matter observables and likelihoods. arXiv: 1705.07920

179. F. Feroz, M.P. Hobson, M. Bridges, MULTINEST: an efficient and robust Bayesian inference tool for cosmology and particle physics. MNRAS 398, 1601-1614 (2009). arXiv:0809.3437

180. J. Brest, S. Greiner, B. Boskovic, M. Mernik, V. Zumer, Selfadapting control parameters in differential evolution: a comparative study on numerical benchmark problems. Evolut. Comput. IEEE Trans. 10, 646-657 (2006)

181. GAMBIT Collider Workgroup, C. Balázs, A. Buckley, et. al., ColliderBit: a GAMBIT module for the calculation of high-energy collider observables and likelihoods. arXiv:1705.07919

182. Planck Collaboration, P.A.R. Ade, et. al., Planck 2015 results. XIII. Cosmological parameters. A\&A 594, A13 (2016). arXiv: 1502.01589

183. Fermi-LAT Collaboration, M. Ackermann, A. Albert, et. al., Searching for dark matter annihilation from milky way dwarf spheroidal galaxies with six years of Fermi large area telescope data. Phys. Rev. Lett. 115, 231301 (2015). arXiv:1503.02641
184. IceCube Collaboration, M.G. Aartsen, R. Abbasi, et. al., Search for dark matter annihilations in the Sun with the 79-string IceCube detector. Phys. Rev. Lett. 110, 131302 (2013). arXiv:1212.4097

185. LUX Collaboration, D.S. Akerib et al., First results from the LUX dark matter experiment at the Sanford Underground Research Facility. Phys. Rev. Lett. 112, 091303 (2014). arXiv: 1310.8214

186. D.S. Akerib, H.M. Araújo et al., Improved Limits on scattering of weakly interacting massive particles from reanalysis of 2013 LUX data. Phys. Rev. Lett. 116, 161301 (2016). arXiv:1512.03506

187. D.S. Akerib, S. Alsum et al., Results from a Search for Dark Matter in the Complete LUX Exposure. Phys. Rev. Lett. 118, 021303 (2017). arXiv: 1608.07648

188. PandaX-II Collaboration, A. Tan et. al., Dark matter results from first 98.7 days of data from the PandaX-II experiment. Phys. Rev. Lett. 117, 121303 (2016). arXiv: 1607.07400

189. C. Amole, M. Ardid et al., Dark matter search results from the

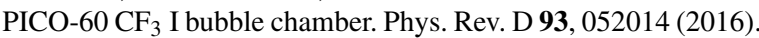
arXiv: 1510.07754

190. PICO Collaboration, C. Amole, et al., Improved dark matter search results from PICO-2L Run 2. Phys. Rev. D 93, 061101 (2016). arXiv: 1601.03729

191. XENON100 Collaboration, E. Aprile, M. Alfonsi, et al., Dark matter results from 225 live days of XENON100 Data. Phys. Rev. Lett. 109, 181301 (2012). arXiv: 1207.5988

192. SuperCDMS Collaboration, R. Agnese et. al., Search for lowmass weakly interacting massive particles with SuperCDMS. Phys. Rev. Lett. 112, 241302 (2014). arXiv: 1402.7137

193. SIMPLE Collaboration, M. Felizardo et. al., The SIMPLE phase II dark matter search. Phys. Rev. D 89, 072013 (2014). arXiv: 1404.4309

194. Particle Data Group. Berkeley, K. Nakamura et al, Review of Particle Properties. J. Phys. G 37, 075021 (2010)

195. G.W. Bennett, B. Bousquet et al., Final report of the E821 muon anomalous magnetic moment measurement at BNL. Phys. Rev. D 73, 072003 (2006). arXiv:hep-ex/0602035

196. GAMBIT Models Workgroup, P. Athron, C. Balázs, et. al., SpecBit, DecayBit and PrecisionBit: GAMBIT modules for computing mass spectra, particle decay rates and precision observables. arXiv: 1705.07936

197. GAMBIT Flavour Workgroup, F. U. Bernlochner, M. Chrzaszcz, et. al., FlavBit: a GAMBIT module for computing flavour observables and likelihoods. arXiv: 1705.07933

198. G. Bélanger, F. Boudjema, A. Pukhov, A. Semenov, micrOMEGAs4.1: two dark matter candidates. Comput. Phys. Commun. 192, 322-329 (2015). arXiv:1407.6129

199. F. Staub, SARAH. arXiv:0806.0538

200. F. Staub, Automatic calculation of supersymmetric renormalization group equations and self energies. Comput. Phys. Commun. 182, 808-833 (2011). arXiv: 1002.0840

201. B.C. Allanach, SOFTSUSY: a program for calculating supersymmetric spectra. Comput. Phys. Commun. 143, 305-331 (2002). arXiv:hep-ph/0104145

202. B.C. Allanach, P. Athron, L.C. Tunstall, A. Voigt, A.G. Williams, Next-to-Minimal SOFTSUSY. Comput. Phys. Commun. 185, 2322-2339 (2014). arXiv:1311.7659

203. P. Athron, J.-H. Park, D. Stöckinger, A. Voigt, FlexibleSUSY—a spectrum generator generator for supersymmetric models. Comput. Phys. Commun. 190, 139-172 (2015). arXiv:1406.2319

204. P. Bechtle, O. Brein, S. Heinemeyer, G. Weiglein, K.E. Williams, HiggsBounds: confronting arbitrary Higgs sectors with exclusion bounds from LEP and the tevatron. Comput. Phys. Commun. 181, 138-167 (2010). arXiv:0811.4169

205. Bechtle P., Brein O., Heinemeyer S., Weiglein G., K.E. Williams, HiggsBounds 2.0.0: confronting neutral and charged Higgs sector predictions with exclusion bounds from LEP and the 
tevatron. Comput. Phys. Commun. 182, 2605-2631 (2011). arXiv: 1102.1898

206. P. Bechtle, O. Brein et al., HiggsBounds-4: improved tests of extended Higgs sectors against exclusion bounds from LEP, the tevatron and the LHC. Eur. Phys. J. C 74, 2693 (2014). arXiv: 1311.0055

207. P. Bechtle, S. Heinemeyer, O. Stål, T. Stefaniak, G. Weiglein, HiggsSignals: confronting arbitrary Higgs sectors with measurements at the Tevatron and the LHC. Eur. Phys. J. C 74, 2711 (2014). arXiv: 1305.1933

208. T. Sjostrand, S. Ask et al., An introduction to PYTHIA 8.2. Comput. Phys. Commun. 191, 159-177 (2015). arXiv:1410.3012

209. F. Mahmoudi, SuperIso: a program for calculating the isospin asymmetry of $B \rightarrow K^{*} \gamma$ in the MSSM. Comput. Phys. Commun. 178, 745 (2008). arXiv:0710.2067

210. F. Mahmoudi, SuperIso v2.3: a program for calculating flavor physics observables in Supersymmetry. Comput. Phys. Commun. 180, 1579 (2009). arXiv:0808.3144

211. F. Mahmoudi, SuperIso v3.0, flavor physics observables calculations: extension to NMSSM. Comput. Phys. Commun. 180, 1718 (2009)

212. A. Djouadi, J. Kalinowski, M. Spira, HDECAY: a program for Higgs boson decays in the standard model and its supersymmetric extension. Comput. Phys. Commun. 108, 56-74 (1998). arXiv:hep-ph/9704448

213. M. Muhlleitner, A. Djouadi, Y. Mambrini, SDECAY: A Fortran code for the decays of the supersymmetric particles in the MSSM. Comput. Phys. Commun. 168, 46-70 (2005). arXiv:hep-ph/0311167

214. A. Djouadi, M.M. Mühlleitner, M. Spira, Decays of supersymmetric particles: the program SUSY-HIT (SUspect-SdecaYHdecay-InTerface). Acta Phys. Polon. 38, 635-644 (2007). arXiv:hep-ph/0609292

215. J.M. Butterworth et. al., The tools and Monte Carlo Working Group summary report from the Les Houches 2009 Workshop on TeV Colliders. In Physics at TeV colliders. Proceedings, 6th Workshop, dedicated to Thomas Binoth, Les Houches, France, June 8-26, 2009 (2010). arXiv: 1003.1643

216. ATLAS Collaboration, Further searches for squarks and gluinos in final states with jets and missing transverse momentum at $\sqrt{s}$ $=13 \mathrm{TeV}$ with the ATLAS detector. Tech. Rep. ATLAS-CONF2016-078 (CERN, Geneva 2016)

217. K.J. de Vries, E.A. Bagnaschi et al., The pMSSM10 after LHC run 1. Eur. Phys. J. C 75, 422 (2015). arXiv: 1504.03260

218. H.K. Dreiner, S. Heinemeyer, et. al., How light can the lightest neutralino be? eConf C0705302, SUS06 (2007). arXiv:0707.1425

219. S. Profumo, Hunting the lightest lightest neutralinos. Phys. Rev. D 78, 023507 (2008). arXiv:0806.2150

220. H.K. Dreiner, S. Heinemeyer et al., Mass bounds on a very light neutralino. Eur. Phys. J. C 62, 547-572 (2009). arXiv:0901.3485

221. A. Arbey, M. Battaglia, F. Mahmoudi, Light neutralino dark matter in the pMSSM: Implications of LEP, LHC and dark matter searches on SUSY particle spectra. Eur. Phys. J. C 72, 2169 (2012). arXiv:1205.2557

222. C. Boehm, P.S.B. Dev, A. Mazumdar, E. Pukartas, Naturalness of light neutralino dark matter in pMSSM after LHC, XENON100 and Planck data. JHEP 06, 113 (2013). arXiv:1303.5386

223. Particle Data Group, C. Patrignani et al., Review of particle physics. Chin. Phys. C 40, 100001 (2016)

224. DELPHI, J. Abdallah et. al., Searches for supersymmetric particles in $e+e-$ collisions up to $208-\mathrm{GeV}$ and interpretation of the results within the MSSM. Eur. Phys. J. C 31, 421-479 (2003). arXiv:hep-ex/0311019

225. L3 Collaboration, M. Acciarri, et al., Search for charginos and neutralin os in $e^{+} e^{-}$collisions at $\sqrt{s}=189 \mathrm{GeV}$. Phys. Lett. B 472, 420-433 (2000). arXiv:hep-ex/9910007
226. M. Misiak, M. Steinhauser, Weak radiative decays of the B Meson and bounds on $M_{H^{ \pm}}$in the two-Higgs-doublet model. Eur. Phys. J. C 77, 201 (2017). arXiv:1702.04571

227. H. Eberl, K. Hidaka, S. Kraml, W. Majerotto, Y. Yamada, Improved SUSY QCD corrections to Higgs boson decays into quarks and squarks. Phys. Rev. D 62, 055006 (2000). arXiv:hep-ph/9912463

228. M. Carena, D. Garcia, U. Nierste, C.E.M. Wagner, Effective Lagrangian for the $\bar{t} b H^{+}$interaction in the MSSM and charged Higgs phenomenology. Nucl. Phys. B 577, 88-120 (2000). arXiv:hep-ph/9912516

229. CMS Collaboration, CMS Moriond 2017 EW Summary Plot. https://twiki.cern.ch/twiki/pub/CMSPublic/PhysicsResultsSUS/ EWKino_limits_summary_cms_Moriond17.pdf

230. CMS Collaboration, CMS Moriond 2017 EW Summary Plot. https://twiki.cern.ch/twiki/pub/CMSPublic/PhysicsResultsSUS/ T2tt_limits_summary_cms_Moriond17.pdf

231. CMS Collaboration, Search for electroweak production of charginos and neutralinos in multilepton final states in pp collision data at $\sqrt{s}=13 \mathrm{TeV}$. Tech. Rep. CMS-PAS-SUS-16-039 (CERN, Geneva, 2017)

232. CMS Collaboration, Search for electroweak production of charginos and neutralinos in the WH final state in proton-proton collisions at $\sqrt{s}=13 \mathrm{TeV}$. Tech. Rep. CMS-PAS-SUS-16-043 (CERN, Geneva, 2017)

233. CMS Collaboration, Search for new physics in final states with two opposite-sign, same-flavor leptons, jets, and missing transverse momentum in pp collisions at $\sqrt{s}=13 \mathrm{TeV}$. Tech. Rep. CMSPAS-SUS-16-034 (CERN, Geneva, 2017)

234. CMS Collaboration, Search for new physics in events with two low momentum opposite-sign leptons and missing transverse energy at $\sqrt{s}=13 \mathrm{TeV}$. Tech. Rep. CMS-PAS-SUS-16-048 (CERN, Geneva, 2017)

235. CMS Collaboration, Search for direct production of bottom and top squark pairs in proton-proton collisions at $\sqrt{s}=13 \mathrm{TeV}$. Tech. Rep. CMS-PAS-SUS-16-032 (CERN, Geneva, 2017)

236. CMS, A.M. Sirunyan et. al., Search for supersymmetry in multijet events with missing transverse momentum in proton-proton collisions at $13 \mathrm{TeV}$. arXiv: 1704.07781

237. CMS Collaboration, Search for new physics in the all-hadronic final state with the MT2 variable. Tech. Rep. CMS-PAS-SUS-16036 (CERN, Geneva, 2017)

238. CMS Collaboration, CMS Moriond 2017 EW Summary Plot. https://twiki.cern.ch/twiki/pub/CMSPublic/PhysicsResultsSUS/ T2bb_limits_summary_cms_Moriond17.pdf

239. M. Schumann, L. Baudis, L. Bütikofer, A. Kish, M. Selvi, Dark matter sensitivity of multi-ton liquid xenon detectors. JCAP 1510, 016 (2015). arXiv:1506.08309

240. DARWIN, J. Aalbers et. al., DARWIN: towards the ultimate dark matter detector. JCAP 1611, 017 (2016). arXiv:1606.07001

241. J. Billard, L. Strigari, E. Figueroa-Feliciano, Implication of neutrino backgrounds on the reach of next generation dark matter direct detection experiments. Phys. Rev. D 89, 023524 (2014). arXiv: 1307.5458

242. CMS Collaboration, Search for direct top squark pair production in the all-hadronic final state in proton-proton collisions at $\sqrt{s}=13 \mathrm{TeV}$. Tech. Rep. CMS-PAS-SUS-16-049 (CERN, Geneva, 2017)

243. CMS Collaboration, Search for top squark pair production in the single lepton final state at $\sqrt{s}=13 \mathrm{TeV}$. Tech. Rep. CMS-PASSUS-16-051 (CERN, Geneva, 2017)

244. CMS Collaboration, Search for direct stop pair production in the dilepton final state at $\sqrt{s}=13 \mathrm{TeV}$. Tech. Rep. CMS-PAS-SUS17-001 (CERN, Geneva, 2017)

245. PICO, C. Amole et. al., Dark matter search results from the PICO$60 C_{3} F_{8}$ bubble chamber. arXiv:1702.07666 
246. C. Amole, M. Ardid et al., PICASSO, COUPP and PICO—search for dark matter with bubble chambers. Eur. Phys. J. Web Conf. 95, 04020 (2015)

247. H. Silverwood, P. Scott et al., Sensitivity of IceCube-DeepCore to neutralino dark matter in the MSSM-25. JCAP 3, 27 (2013). arXiv: 1210.0844

248. KM3NeT Collaboration, S. Adrian-Martinez et. al., Letter of intent for KM3NeT 2.0. J. Phys. G 43, 084001 (2016). arXiv: 1601.07459

249. IceCube PINGU Collaboration, M.G. Aartsen et. al., Letter of intent: the precision IceCube next generation upgrade (PINGU). arXiv: 1401.2046

250. IceCube Collaboration, M.G. Aartsen et. al., IceCube-Gen2: a vision for the future of neutrino astronomy in Antarctica. arXiv: 1412.5106

251. LUX Collaboration, D.S. Akerib et. al., Limits on spin-dependent WIMP-nucleon cross section obtained from the complete LUX exposure. arXiv: 1705.03380

252. Fermi-LAT Collaboration, E. Charles et. al., Sensitivity projections for dark matter searches with the Fermi large area telescope. Phys. Rep. 636, 1-46 (2016). arXiv: 1605.02016

253. CTA Consortium, J. Carr et. al., Prospects for indirect dark matter searches with the Cherenkov telescope array (CTA). PoS ICRC2015, 1203 (2016). arXiv:1508.06128

254. A. Djouadi, M. Drees, QCD corrections to neutralino nucleon scattering. Phys. Lett. B 484, 183-191 (2000). arXiv:hep-ph/0004205
255. A. Djouadi, M. Drees, P. Fileviez Perez, M. Muhlleitner, Loop induced Higgs and $\mathrm{Z}$ boson couplings to neutralinos and implications for collider and dark matter searches. Phys. Rev. D 65, 075016 (2002). arXiv:hep-ph/0109283

256. H. Silverwood, C. Weniger, P. Scott, G. Bertone, A realistic assessment of the CTA sensitivity to dark matter annihilation. JCAP 1503, 055 (2015). arXiv:1408.4131

257. L. Bergstrom, P. Ullio, Full one loop calculation of neutralino annihilation into two photons. Nucl. Phys. B 504, 27-44 (1997). arXiv:hep-ph/9706232

258. T. Bringmann, L. Bergström, J. Edsjö, New gamma-ray contributions to supersymmetric dark matter annihilation. JHEP 01, 049 (2008). arXiv:0710.3169

259. T. Bringmann, F. Calore, A. Galea, M. Garny, Electroweak and Higgs boson internal Bremsstrahlung: general considerations for Majorana dark matter annihilation and application to MSSM neutralinos. arXiv: 1705.03466

260. J. Hisano, S. Matsumoto, M.M. Nojiri, Unitarity and higher order corrections in neutralino dark matter annihilation into two photons. Phys. Rev. D 67, 075014 (2003). arXiv:hep-ph/0212022

261. J. Hisano, S. Matsumoto, M.M. Nojiri, O. Saito, Non-perturbative effect on dark matter annihilation and gamma ray signature from galactic center. Phys. Rev. D 71, 063528 (2005). arXiv:hep-ph/0412403

262. A. Hryczuk, R. Iengo, P. Ullio, Relic densities including Sommerfeld enhancements in the MSSM. JHEP 03, 069 (2011). arXiv: 1010.2172 